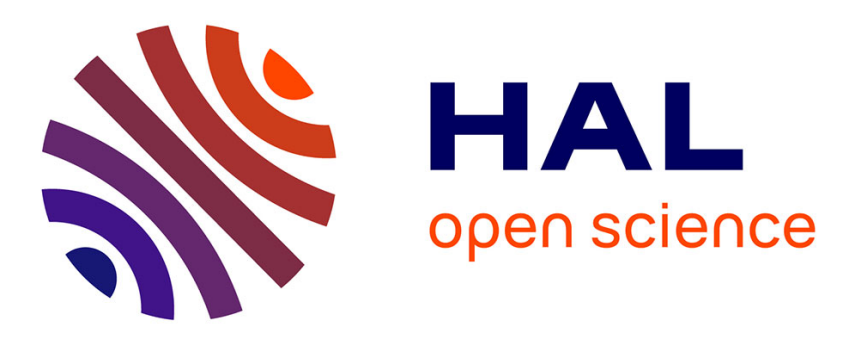

\title{
Separation of SSR Signals by Array Processing in Multilateration Systems
}

\author{
Nicolas Petrochilos, Gaspare Galati, Emilio Piracci
}

\section{To cite this version:}

Nicolas Petrochilos, Gaspare Galati, Emilio Piracci. Separation of SSR Signals by Array Processing in Multilateration Systems. IEEE Transactions on Aerospace and Electronic Systems, 2009, 45 (3), pp.965 - 982. 10.1109/TAES.2009.5259177 . hal-01693613

\section{HAL Id: hal-01693613 \\ https://hal.univ-reims.fr/hal-01693613}

Submitted on 7 Dec 2019

HAL is a multi-disciplinary open access archive for the deposit and dissemination of scientific research documents, whether they are published or not. The documents may come from teaching and research institutions in France or abroad, or from public or private research centers.
L'archive ouverte pluridisciplinaire $\mathbf{H A L}$, est destinée au dépôt et à la diffusion de documents scientifiques de niveau recherche, publiés ou non, émanant des établissements d'enseignement et de recherche français ou étrangers, des laboratoires publics ou privés. 


\title{
Separation of SSR Signals by Array Processing in Multilateration Systems
}

\author{
Nicolas Petrochilos, Gaspare Galati, and Emilio Piracci \\ DISP and V. VOLTERRA CENTER, \\ Tor Vergata University, \\ Via del Politecnico, 1, 00133 Roma, Italy \\ petro@ieee.org, g.galati@ieee.org, piracci@disp.uniroma2.it
}

KEYWORDS: Secondary Surveillance Radar, Mode S, Multilateration Systems, Air Traffic Management Airport Surface Traffic, Runway Incursion, Array Signal Processing, Source separation

\begin{abstract}
Location and identification of cooperating aircraft in the airport area (and beyond) may be implemented by multilateration (MLAT) systems using the Secondary Surveillance Radar (SSR) Mode S signals. Most of these signals, spontaneously emitted from onboard Mode $\mathrm{S}$ transponders at a fixed carrier frequency, arrive randomly at the receiving station, as well as many Mode A/C replies from legacy transponders still in use. Several SSR signals are, then, overlapped in multiple aircraft situations. Therefore, the aim of this work is the separation of overlapped SSR signals, i.e. signals superimposed in time at receiving stations. We improve the MLAT receiving station by replacing the single antenna by an array of $m$ elements and using array signal processing techniques. In the literature, several algorithms address the general source separation problem, but a very few of them are specifically designed for a mixture of overlapping SSR replies. Unfortunately, all of them have either some shortcomings, or an expensive computational cost, or no simple practical implementation. In this paper, we use the time sparsity property of the sources to propose more reliable, simpler, and more effective algorithms based on projection techniques to separate multiple SSR signals. Real recorded signals in a live environment are used to demonstrate the effectiveness of our method.
\end{abstract}

\section{INTRODUCTION}

Originally denominated "Identification Friend or Foe" (IFF) during the Second World War [1], the Secondary Surveillance Radar (SSR) is becoming a fundamental tool for Communication and Surveillance in the Air Traffic Management (ATM) and Airport Surface Movements Guidance and Control (SMGCS). Indeed, the Mode $\mathrm{S}$ data link has been chosen by the Federal Aviation Administration (FAA) of the USA in 2002 as the standard transmission means for carrier aircraft.

Aircraft -and surface vehicles- detection, identification, tracking, and communication by the use of Multilateration systems based on SSR, as shown in Figure 1 , is expanding dramatically both for airport surface [2], [3], and in their Wide Area version [4], [5], due to their improved performances. But various users of the SSR channel (at $1090 \mathrm{MHz}$ ) do exist or are planned: (a) the classical SSR (Mode A/C, Mode S), (b) the Multilateration, (c) the Automatic Dependent Surveillance - Broadcast (for short, ADS-B) in which on board navigation data are periodically transmitted to a surveillance central facility through a network of distributed receiving stations, and (d) the Traffic Infor-

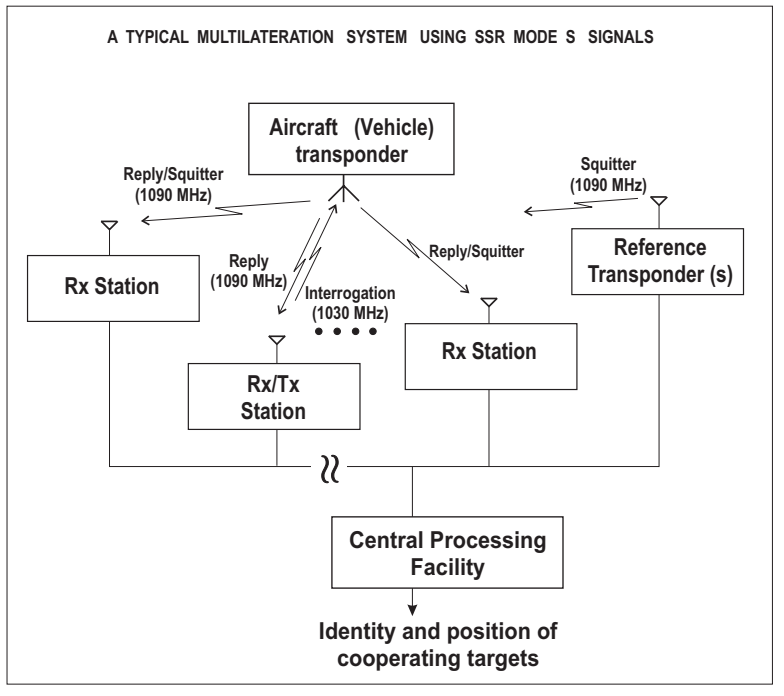

Fig. 1. The distributed SSR (multilateration) system.

mation Service - Broadcast (TIS-B) which transmits to aircraft and airport vehicles the traffic situation (position, identity of targets of interest in a given coverage area). As the receiving stations use omnidirectional (more precisely, wide beam) antennae, the number of received signals per time unit increases with respect to the classical SSR ground stations with narrow beam antennae. With conventional receivers with a single antenna, when the replies are overlapped ("garbled"), very often the message transmitted by the aircraft is corrupted and cannot be recovered, nor can the aircraft be located and identified.

We propose to circumvent this problem by using an antenna array that, in front of the obvious increase of cost and complexity - with respect to the classical single-antenna systems - permits to separate (or de-"garble") the estimated number of replies and to send each estimated reply to a different conventional receiver, see Fig. 2.

Source separation algorithms use either array response properties or source properties. Source separation based on the array response matrix (for instance $[6],[7],[8])$ is not reliable in our application, as it needs a perfect calibration and does not tolerate multipath. 


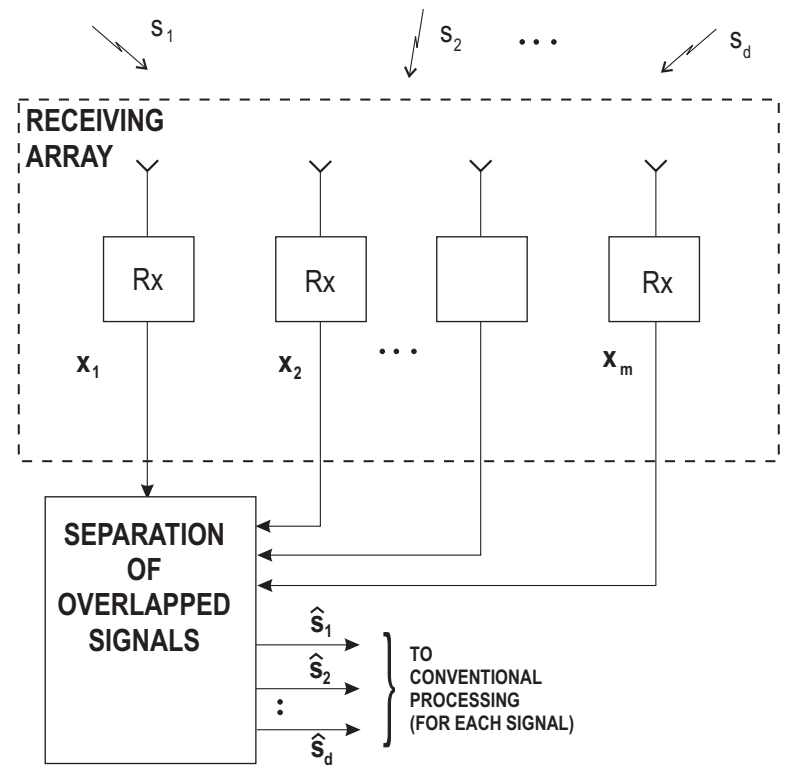

Fig. 2. Sketch of proposed receiver: a processor separates (unmixes) the incoming replies to send each of them to an individual conventional receiver consisting of a preamble detector and a decoder.

High-Order Statistic Separation algorithms [9], [10], [11] can't be used, as the replies are "pseudo-Gaussian" up to order 5 [12], [13], i.e. all cumulants of order 3,4 and 5 have a large probability to be very close to zero. Even Fast-ICA and later versions [14] do not work well because a large number of samples (of the order of a few thousand) is needed to exploit the statistical independence. Deterministic separation algorithms based on the very structured but unusual source model have been considered in [15], [5], [16], but they separate only mixtures of Mode S, they have a high computational burden, and their final step is a joint diagonalization of several 3-rd or 4-th order tensors [17]. The best one, the Manchester Decoding Algorithm (MDA) [16], has been demonstrated not to properly work in the case of small overlaps in time (i.e. large relative delays). Moreover, these deterministic algorithms have been designed for Mode $\mathrm{S}$, and their pulse lengths are incommensurate with Mode A/C pulse lengths, so they fail in the presence of Mode A/C. A second-order algorithm based on colored sources [18] has been shown to under-perform the deterministic algorithm in [5], due to the high energy level of off-diagonal terms in the auto-correlation matrices.

In this paper, we focus on the blind separation of a mixture of SSR replies. We use the sparsity in the time domain of the SSR replies to develop two algorithms [19], [20]; the principle of sparsity is to transform the data into a different representation where its description is sparse: for instance it can be the frequency domain, a wavelet description, or even the time domain, see [21], [22], [23]. In the rest of this paper, the first one is presented to enlighten the principle of operation, and deals with the problem of two Mode S replies overlapping in time; the second one is more general in scope.
It allows the separation of any kind of mixture of Mode $\mathrm{A} / \mathrm{C} / \mathrm{S}$ under mild conditions and works on a principle of deflation by projection, and can handle the present mixed situation as well as the future ones, including the simultaneous presence of "long" and "short" Mode $\mathrm{S}$ signals.

The analysis of the proposed algorithm is done using real-world SSR signals, received and recorded by means of an ad-hoc system implemented and owned by the Technical University of Delft [5]. This system is made up by a four-elements receive array connected to a wide band digital acquisition system; thanks to it, a large amount (hundreds) of signals has been recorded in the summer of 2003 [24].

This section continues with additional background knowledge. In the following section, we first present the data model, the problem investigated and the actual multilateration receivers. Next, in section III, the algorithms are described and discussed, while in section IV the experimental data is used to evaluate our algorithms, the evaluations are commented and conclusions are drawn.

\section{A. SSR, short description}

SSR operates on an interrogation-reply basis (unlike the even older "primary" radars based on echo location). The SSR ground station emits an interrogation, eliciting from the cooperating aircraft in the illuminating beam a reply generated by an on-board SSR transponder. The reply signals are pulse-position modulated finite-length signals at the nominal carrier frequency of $1090 \mathrm{MHz}$ [1]. Two operational Modes currently co-exist: the conventional un-addressed Mode $\mathrm{A} / \mathrm{C}$ and the newer Mode $\mathrm{S}$, in which the ground station selectively addresses the aircraft and permits short data communications between the ground interrogating station and the aircraft [1], [25]. This new standard, intended to reduce the interrogation and reply rate, will ultimately replace the Mode A/C. The SSR Mode S interrogation and reply signals are used in the Airborne Collision Avoidance System (ACAS) presently mandatory on all passengers aircraft above a given capacity. Additionally, and very importantly, the Mode S transponders emit spontaneous replies of a similar format named "squitter".

SSR Mode S signals, in their "long"(i.e. 112 bit) format permit air-to-ground and ground-to-air data transmissions that add value to the traditional surveillance using short (56 bit) messages. Note that both ADS-B (Automatic Dependent Surveillance, Broadcast) and TIS-B (Traffic Information Service, Broadcast) use the message format called $1090 \mathrm{ES}$, which stands for $1090 \mathrm{MHz}$ Extended Squitter.

\section{B. Multilateration System, short description}

Multilateration systems (both "short range" for airport coverage and "wide area"), as previously described, are basically distributed surveillance and identification systems, (Figure 1). They are made up of a 
network of some (e.g. ten to fifteen) receiving stations or "sensor" stations (a part of them, e.g. one third, also has interrogation capabilities), one or a few reference transponders in geo-referenced positions to synchronize and calibrate the whole sensor system, a communication medium (typically, a Local Area Network) and a Central Processor where location (multilateration) algorithms run for the multisensor fusion. These algorithms rely on the estimation of the Time of Arrival (ToA) at the stations where the target is visible to perform intersection of many hyperbolic surfaces, as obtained by Time Difference of Arrival (TDoA) techniques [26].

\section{Receivers}

Conventional receivers with a single antenna are well known, as they can be found in present days airports. They work on a two-step basis: preamble detection (with time synchronization for decoding of message bits), then message decoding. However, today's "degarbling" algorithms work only when the degree of overlapping is very limited.

A simple evaluation of the expected congestion problem on the $1090 \mathrm{MHz}$ channel can be obtained [24] considering a number $N$ of transponders (on board aircraft and airport vehicles) in an airport randomly transmitting one short $(64 \mu \mathrm{s})$ and one long $(120 \mu \mathrm{s})$ Mode $\mathrm{S}$ signal (squitter) at an average rate of one or two per second. In order to get a detection probability of $95 \%$ every second with a conventional receiving Multilateration station, with no degarbling capability, $N$ has to be less than 121 (and less than 24 for $99 \%$ detection probability). With a new station capable of detecting and decoding two overlapped squitters or replies, the maximum number of transponders, $N$, increases to 2284 (and to 984 for $99 \%$ probability): these values are greater than the maximum number of expected active transponders in the airport. Problems of SSR traffic in terminal areas and the need for more modern processing techniques have been treated in [27].

In order to guarantee better performance, it is possible either to exploit the variation of the center frequency of the on-board transponder [28] (it is due to tolerance, therefore it is rather limited) or to use an array of several antenna elements instead of a single antenna, as proposed here. Note that the use of an array allows not only source separation, but also Direction of Arrival (DoA) estimation for each source. DoA estimation needs array calibration; this paper only deals with separation that has no need for calibration.

\section{DATA MODEL}

\section{A. Notation}

We denote scalars by italic lowercase letters, as in $a$, vectors by lower case boldface letters, as in $\mathbf{a}$, and matrices by upper case boldface letters, as in A. Unless specified otherwise, $\mathbf{I}$ is the identity matrix, and $\mathbf{0}$ and $\mathbf{1}$ are the vectors with all entries are equal to 0 and 1 , respectively. We denote by $(.)^{*}$ the complex conjugation, by $(.)^{T}$ the matrix transpose, and by $(.)^{H}$ the matrix conjugate transpose.

$\mathrm{E}\{$.$\} denotes the statistical expectation operator.$ $|\mathbf{A}|$ refers to the determinant of the square matrix $\mathbf{A}$. The notation $(.)^{\dagger}$ refers to the Moore-Penrose inverse (pseudo-inverse)[29], [30]. For tall matrices, if $\mathbf{A}^{H} \mathbf{A}$ is invertible, then: $\mathbf{A}^{\dagger}=\left(\mathbf{A}^{H} \mathbf{A}\right)^{-1} \mathbf{A}^{H}$, and $\mathbf{A}^{\dagger} \mathbf{A}=\mathbf{I}$. Within a matrix, the sign $*$ denotes a scalar or a submatrix of non-relevant value.

\section{B. Model}

The SSR transmissions from air-to-ground and from ground-to-air are (Sect. I) Mode A/C and Mode S. For the sake of simplicity, we do not include the full details of the transmitted signals that are explained $i n$ ter alia, in [1] and thoroughly specified in the "Annex 10" of the Convention on the International Civil Aviation, [31]. Here we are only interested in air-to-ground, or downlink transmission, where both Mode $\mathrm{A} / \mathrm{C}$ and Mode S signals are pulse-modulated (the modulation depending on the Mode) and of limited time duration. Thus they are packet-wise of different lengths, resp. $21.7 \mu$ s and $64 / 120 \mu$ s (i.e. Short/Long Mode S reply), with different pulse lengths.

For the sake of simplicity, let us just consider it as a binary signal denoted $b[n]$, when sampled at rate $T_{e}$. Before being emitted by the antenna, the signal is up-converted to the frequency $f_{e}$. Nominally, the carrier frequency is $f_{c}=1090 \mathrm{MHz}$, but the tolerance originally permitted by the ICAO [31] is up to $\pm 3 \mathrm{MHz}$ (presently reduced to $\pm 1 \mathrm{MHz}$ ), thus $f_{e} \neq f_{c}$. Due to this carrier frequency mismatch, a residual frequency $f$ remains after down-conversion by $f_{c}$ to baseband. The baseband antenna signals are sampled at a frequency greater than the signal bandwidth (in our experiments the sampling rate is $T_{e}=0.02 \mu \mathrm{s}$ ). The residual carrier $f$ adds a significant phase rotation to the transmitted symbols: up to a complex gain, the received baseband signal $s[n]=s\left(n T_{e}\right)$ is described as

$$
s[n]=b[n] \exp \left(\jmath 2 \pi n f T_{e}\right)=b[n] \phi^{n}
$$

where $\phi=\exp \left(\jmath 2 \pi f T_{e}\right)$ is the phase shift due to the carrier frequency shift over a sampling period.

We consider the reception of $d$ independent source signals on an $m$-element antenna array (of arbitrary form). The baseband antenna signals, the $x_{i}[n]$ 's with $i \in[1, . ., m]$, are stacked in vectors $\mathbf{x}[n]($ size $m)$. After collecting $T$ samples, the observation model is

$$
\mathbf{X}=\mathbf{M} \cdot \mathbf{S}+\mathbf{N}
$$

where $\mathbf{X}=[\mathbf{x}[1], \cdots, \mathbf{x}[T]]$ is the $m \times T$ received signal matrix. $\mathbf{S}=[\mathbf{s}[1], \cdots, \mathbf{s}[T]]$ is the $d \times T$ source matrix, where $\mathbf{s}[n]=\left[s_{1}[n], \cdots, s_{d}[n]\right]^{T}$ is a stacking of the $d$ source signals. $\mathbf{N}$ is the $m \times T$ noise matrix, whose elements are temporally and spatially white. $\mathbf{M}$ is the $m \times d$ mixing matrix that contains the array signatures and the complex gains of the sources. Figure 3 presents a typical real case of mixed replies, where 


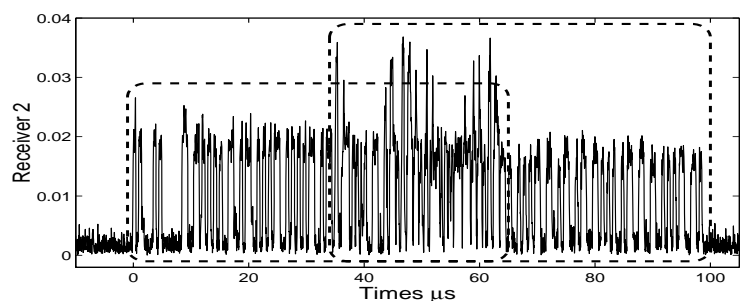

Fig. 3. A record of overlapped replies (case W5), [31].

actually two Mode S's (in boxes) and one Mode A/C (merely visible) are present.

We assume that the replies are independent (hence: uncorrelated), i.e. $\mathrm{E}\left\{s_{i} s_{j}^{*}\right\}=0$ for $i \neq j$, that $d \leq m$ and $\mathbf{M}$ is full column rank.

Assuming no multipath, the matrix $\mathbf{M}$ contains the array signatures and the complex gains of the sources: $\mathbf{M}=\mathbf{A G}$. Let us consider a linear array where $\Delta_{k}$ is the distance between the $k$-th element and the first element $(1 \leq k \leq m)$ and $f_{i}$ the frequency of the $i$ th source (remember that $f_{i}$ is slightly different from $\left.f_{c}\right)$. A is the $m \times d$ steering matrix that contains the $m$-elements steering vectors $\mathbf{a}\left(\theta_{i}\right), 1 \leq i \leq d$ defined as:

$$
\mathbf{a}\left(\theta_{i}\right)=\left[\begin{array}{c}
1 \\
\exp \left(\jmath \frac{2 \pi f_{i}}{c} \Delta_{2} \sin \left(\theta_{i}\right)\right) \\
\vdots \\
\exp \left(\jmath \frac{2 \pi f_{i}}{c} \Delta_{m} \sin \left(\theta_{i}\right)\right)
\end{array}\right]
$$

We considered during the simulations a uniform linear array (ULA) of 4 elements with the inter-distance between the elements chosen as $\Delta=\frac{\lambda}{2}$, and all sources at nominal frequency $f_{c}$ :

$\mathbf{a}\left(\theta_{i}\right)=\left[1, \exp \left(\jmath \pi \sin \left(\theta_{i}\right)\right), \cdots, \exp \left(\jmath \pi \sin \left(\theta_{i}\right)\right)^{(m-1)}\right]^{T}$

where $\theta_{i}$ are the directions of incidence of the $i$-th source with respect to the ULA boresight direction.

Note that $\mathbf{M}$ can be considered as an unparameterized matrix that can also reflect multipath, the imperfections of the array such as calibration errors, coupling errors, or inaccuracies in the position of the elements. Considering that $m>d$, we only assume the matrix $\mathbf{M}$ to have full column rank and so to be left-invertible.

\section{Conventional receiver}

In section IV-A, we compare our algorithm over real data to other source separation algorithms, and to an actual SSR system. Such a system is made up by an analog section (antenna and radio frequency/intermediate frequency receiving chain), a digital section (signal processing), and a plot extractor. Secondary Radar systems use a rotating, shaped-beam LVA (Large Vertical Array), while MLAT stations are equipped by a simple, wide-beam antenna such as a vertical dipole. The conventional signal processing consists of two steps: "signal extraction" and decoding. Generally the signal extraction produces a one bit (presence/absence) signal, obtained by (a) comparing the input signal amplitude with a threshold and (b) comparing length and position of pulses with the standard values [1], [31]. This "presence" signal is used to perform detection and decoding of the whole signal. The detection of particular pulses supplies the timing synchronization. For SSR Mode A/C replies, the time synchronization is done by "bracket" detection, while for SSR Mode S it is done by preamble detection. Next to it, the decoding section, declares the bit code using the signal amplitude, the presence signal, and the estimated time reference. Downstream the "signal extraction", the decoding algorithms perform the recognition of a "valid" reply and its decoding. Here we only consider the Mode $\mathrm{S}$ replies, that are modulated by PPM (Pulse Position Modulation) technique. Therefore, the decoding of each one of the 56 or 112 bits that make up the data block is done by analyzing the pertaining $1 \mu \mathrm{s}$ long-chip, composed of two $0.5 \mu$ s sub-chips (see the upper part of Figure 4 ). If the amplitude in the leading half of the long-chip is greater than in the trailing half, the bit is decoded as 1 logical, 0 logical otherwise. To perform the error check and correction, the receiver establishes the confidence level of each bit. The criteria to establish the decoded bit confidence are based on two elements: a noise threshold and a $\pm 1.5 \mathrm{~dB}$ amplitude interval centered on the preamble pulses level. A Low Confidence bit is declared if both the levels of the samples in the middle of the bit sub-chip are greater than the noise threshold and at the same time they do not belong to the $\pm 1.5 \mathrm{~dB}$ preamble interval or both are lower than the noise threshold. The knowledge of the ICAO 24 bit aircraft identity code is essential to obtain the error correction. Only squitters, recognized from the Downlink Format (DF) field $(\mathrm{DF}=11)$, contain the ID code in the last 24 bit [31], and can be directly corrected. Finally, based on detected, decoded and error corrected replies, the plot extractor provides synthetic information, i.e. a plot, for each detected SSR target and for each data renewal interval (antenna revolution period or equivalent time interval). The plots, possibly fused with other sensors (e.g. primary radar) are used to refresh the aircraft tracks and to show to the ATC operator the scenario on a monitor. An evolution of the conventional receiver can be obtained by the use of matched filter for the preamble detection and for replies decoding, and by the use of super resolution algorithms to decode overlapped bits. For the preamble detection, the signal envelope is sent to the input of the filter matched to the Mode S preamble and to a differentiator. From the zero crossing position it is possible to precisely estimate the position of the preamble pulses, i.e. the ToA of the reply. For replies decoding we compare the amplitude of the pulse matched filter outputs (the filter is matched to the Mode S pulse) in 
each chip of the reply. Using the preamble matched filter and the pulse matched filter, we improve detection probability, number of Low Confidence bits and number of errors (wrong bits) in the decoded reply [24].

\section{Algorithms}

This work aims at separating the SSR replies, by deriving the beamformers $\mathbf{w}_{i}, i=1, \cdots, d$, such that:

$$
\hat{s}_{i}[n]=\mathbf{w}_{i}^{H} \mathbf{x}[n], \quad n=1,2, \ldots, T
$$

is an estimate of the $i$-th SSR signal. In this blind source separation context, we can only try to ensure that each $\hat{s}_{i}[n]$ behaves as an SSR signal, and that the collection of signal estimates is independent.

Toward this goal, we have derived two algorithms: the Projection Algorithm (PA) that is effective to separate two Mode S replies, and the Extended Projection Algorithm (EPA), which aims at separating a more general kind of mixture. The behavior of each algorithm is shown by studying real cases.

\section{A. Projection Algorithm}

We consider here the case of two overlapped Mode S signals (see Figure 4). The case of an overlap between a Mode S and a Mode A/C has been taken care of by the original signals definition and the related system design (see [1]), but only to recover the Mode S reply. In order to recover both replies, the EPA algorithm from the next section has to be used. The algorithm is designed for the practical case when two Mode $\mathrm{S}$ replies have significantly different times of arrival, $t_{1}$ and $t_{2}$, and whose difference is not necessarily an integer multiple of $0.5 \mu \mathrm{s}$. The time support ${ }^{1}$ of the two sources is then partly overlapping, in such a way that at the beginning of the data record $\left(t_{1}\right.$ till $\left.t_{2}\right)$ there is only one source present; while at the end of the data $\left(t_{3}\right.$ till $\left.t_{4}\right)$, only the other source is present (see for instance Figure 3).

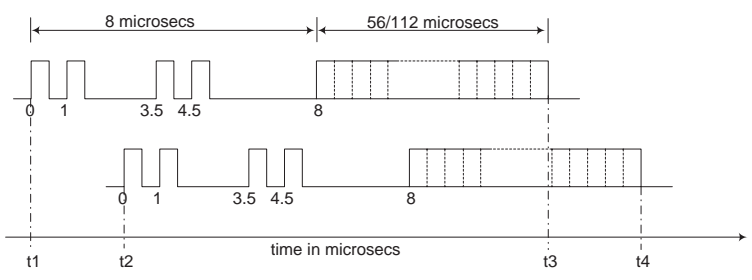

Fig. 4. Two Mode S overlapped replies.

The algorithm begins with the detection of the $t_{i}$ 's. The data is sliced by time slots of 200 samples $(4 \mu \mathrm{s})$. This duration has been chosen long enough to include at least two pulses (see Figure 4) for the whole signal, including the preamble. A whiteness test [32] based on the singular value decomposition (SVD [30]) is performed on each time slot, see Figure 5. This allows us to estimate the number of sources as a function of time, and to isolate the two time supports where each source is single, i.e. $t_{1} \div t_{2}$ and $t_{3} \div t_{4}$ in Figure 5 .

\footnotetext{
${ }^{1}$ By time support, we mean the interval of time from the start of the signal to its end.
}

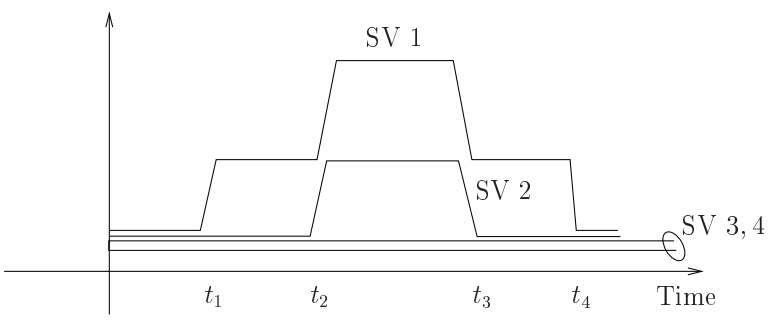

Fig. 5. The singular values, from the SVD, as a function of time.

Be the notation $(.)^{(1)}$ the matrix collecting the subset of the columns related to the interval $t_{1}$ till $t_{2}$ (selection of the columns). Similarly, we define the notation $(.)^{(2)}$ for the subset of the columns related to the interval $t_{3}$ till $t_{4}$. Then, from equation (2), we have the following relation:

$$
\begin{aligned}
& \mathbf{X}^{(1)}=\mathbf{M} \cdot \mathbf{S}^{(1)}+\mathbf{N}^{(1)} \\
& \mathbf{X}^{(2)}=\mathbf{M} \cdot \mathbf{S}^{(2)}+\mathbf{N}^{(2)}
\end{aligned}
$$

where the matrix $\mathbf{S}^{(1)}$ is the $\left(d \times T^{(1)}\right)$ sub-matrix of $\mathbf{S}$ containing the samples emitted in the time interval $\left[t_{1}, t_{2}\right]$, when $T^{(1)}$ is the number of time samples, similarly for $\mathbf{S}^{(2)}, T^{(2)}$, and the time interval $\left[t_{3}, t_{4}\right]$.

$\mathbf{X}^{(1)}$ is then the received $\left(m \times T^{(1)}\right)$ sub-matrix, and $\mathbf{N}^{(1)}$ the receiver noise contribution (resp. for $\mathbf{X}^{(2)}$ and $\mathbf{N}^{(2)}$ ). By construction, $\mathbf{X}^{(1)}$ contains only the first source and can be simplified as (resp. for $\mathbf{X}^{(2)}$ ):

$$
\begin{aligned}
& \mathbf{X}^{(1)}=\mathbf{m}_{1} \cdot \mathbf{s}_{1}^{(1)}+\mathbf{N}^{(1)} \\
& \mathbf{X}^{(2)}=\mathbf{m}_{2} \cdot \mathbf{s}_{2}^{(2)}+\mathbf{N}^{(2)}
\end{aligned}
$$

where the $\mathbf{m}_{i}$ 's are the columns of $\mathbf{M}$, and the $\mathbf{s}_{i}$ 's the rows of $\mathbf{S}$.

Note that $\mathbf{X}^{(1)}$ and $\mathbf{X}^{(2)}$ are rank-one matrices in the noiseless case. By a Singular Value Decomposition (SVD) of $\mathbf{X}^{(1)}$ (resp. $\mathbf{X}^{(2)}$ ), we can estimate the main vector $\hat{\mathbf{m}}_{1}$ (resp. $\hat{\mathbf{m}}_{2}$ ) as the vector corresponding to the greatest singular value.

Once the space signatures $\hat{\mathbf{m}}_{1}$ and $\hat{\mathbf{m}}_{2}$ have been identified, the $(m \times 2)$ matrix $\widehat{\mathbf{M}}$ is thus immediately estimated. The space filters $\mathbf{w}_{i}$ 's are the rows of the Moore-Penrose pseudo-inverse of $\widehat{\mathbf{M}}$. Therefore the $\mathbf{w}_{i}$ 's are orthogonal to the $\mathbf{m}_{j}$ 's if $i \neq j$. In a real two-dimensional space, the space filters $\mathbf{w}_{i}$ can be interpreted as the orthogonal projection of $\hat{\mathbf{m}}_{i}$, parallel to $\hat{\mathbf{m}}_{j}(j \neq i)$, see Figure 6 .

If the angle between $\mathbf{m}_{1}$ and $\mathbf{m}_{2}$ tends towards zero, then the mixing matrix is ill-conditioned, and the beamformer will decrease the signal-to-noise ratio. The limitation on the angle is evidenced in the Appendix for a uniform linear array.

Downstream of our separation algorithm, the extracted replies have to be decoded. To this end, we use a conventional decoder (or better, an improved one based on matched filtering), as described in subsection II-C.

Remark: Our proposed algorithm cannot separate totally overlapping replies, therefore another algorithm 


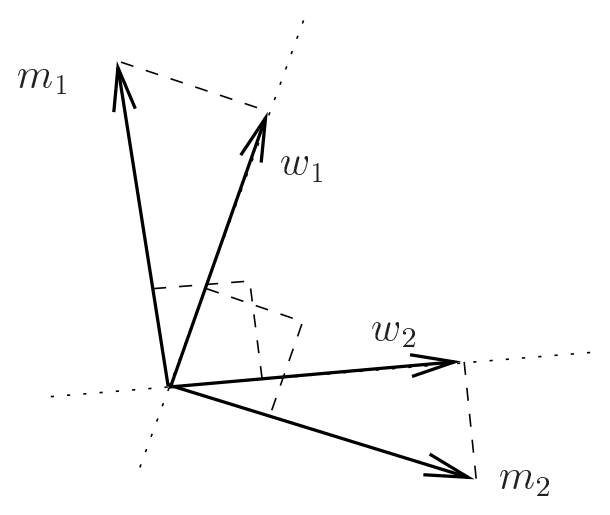

Fig. 6. Principle of the orthogonal Projection.

has to be used, as, for instance, the Manchester Decoding Algorithm [16] which is dedicated to this special case, but more expensive computationally, and more tricky to implement on hardware. The choice of which algorithm has to be used can be done automatically by comparing the difference of time of arrival $\Delta t=t_{2}-t_{1}$, with a pre-determined threshold.

\section{B. Extended Projection Algorithm (EPA)}

The PA algorithm can estimate only two array signatures $\mathbf{m}_{i}$ 's: one at the beginning of the data block, the other at the end. Therefore, it is not possible to separate three or more sources, nor one source including another, e.g. as shown in Figure 7.

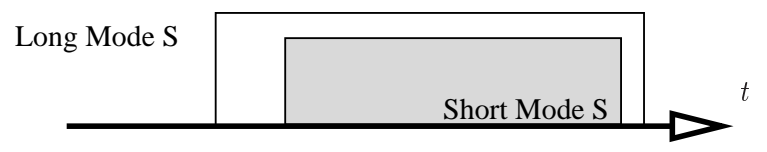

Fig. 7. Case of a Short Mode S included in a Long one.

Figure 8 presents such a case where 4 replies are received, and where only the array signature estimation of the leading Mode S reply seems realistic with the PA.

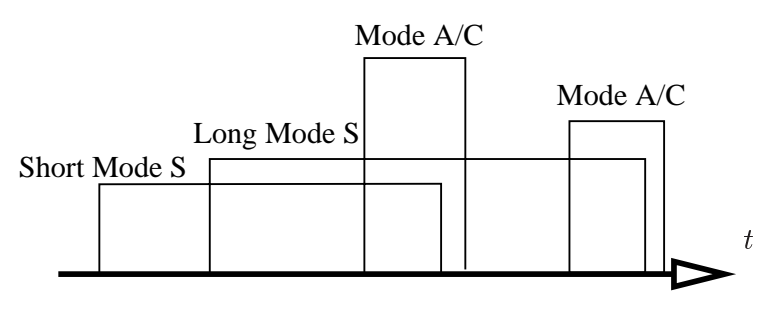

Fig. 8. Case of multiple received replies.

In this section, we describe a method to remove one reply from the mixture; its recursive application, by deflation, separates all replies one by one.

We consider now a mixture of several replies encoded in any Mode. Assuming again that $d \leq m$, we first reduce the received signal dimension $m$ to $d$ by projecting $\mathbf{X}$ onto the signal subspace $\mathbf{V}_{d}$. This subspace is obtained by a SVD, in which the $d$ right singular vectors that corresponds to the $d$ largest singular values are chosen because this subspace correspond to the signal subspace [33]. Then:

$$
\mathbf{V}_{d}=\widetilde{\mathbf{M}}_{d} \mathbf{S}+\widetilde{\mathbf{N}}
$$

with $\widetilde{\mathbf{M}}_{d}$ a reduced $d \times d$ square mixing matrix. From now on, we drop the tilde, (.), from the notation.

The next proposition allows the removal of a source from the received signal:

Proposition III.1: Given $d$ overlapping signals received by a reduced $d$-element array with $T$ time samples, with the noiseless model: $\mathbf{V}_{d}=\mathbf{M}_{d} \mathbf{S}$. Assuming that (A1) $\mathbf{M}_{d}$ is a $d \times d$ full rank matrix (and practically having a good conditioning number), (A2) one source of the mixture is present alone over a substantial time slot $\Delta T$ such that it is possible to detect it, (A3) this source is orthogonal to the other sources, (A4) $\mathbf{S}$ is a $d \times T$ full rank matrix, then:

$\alpha$ ) under (A1-2) a signal subspace of dimension $d-1$ can be extracted, which contains only the $d-1$ remaining signals. $\beta)$ adding $(\mathbf{A} \mathbf{3}-\mathbf{4})$ permits the recovery of this one source.

Proof: We describe the algorithm as a proof. Using (A2), by a rank-one decomposition, we estimate the signature vector $\mathbf{m}_{1}$ of the one reply present over $\Delta T$. We construct the matrix $\mathbf{P}$ to be a basis for the vector space $\mathbb{C}$, where the first vector is $\frac{\mathbf{m}_{1}}{\left\|\mathbf{m}_{1}\right\|}(\|\cdot\|$ stands for the Euclidean norm of a vector):

$$
\mathbf{P}=\left[\begin{array}{lllll}
\frac{\mathbf{m}_{1}}{\left\|\mathbf{m}_{1}\right\|} & \mathbf{w}_{2} & \mathbf{w}_{3} & \ldots & \mathbf{w}_{d}
\end{array}\right]
$$

where by construction, $\forall i \in\{2, d\} \mathbf{w}_{i}^{H} \mathbf{m}_{1}=0$.

Denote $\mathbf{P}_{d-1}$ the sub-matrix containing only the last $d-1$ columns of $\mathbf{P}$. Then the resulting product $\mathbf{V}_{d-1}=\mathbf{P}_{d-1}^{H} \mathbf{V}_{d}$ is a $(d-1) \times T$ matrix that contains only the sources 2 till $d$, such as (in the noiseless case)

$$
\mathbf{V}_{d-1}=\mathbf{P}_{d-1}^{H} \mathbf{V}_{d}=\mathbf{P}_{d-1}^{H} \mathbf{M}_{d} \mathbf{S}
$$

Note that by construction:

$$
\begin{aligned}
\mathbf{P}_{d-1}^{H} \mathbf{M}_{d} & =\left[\begin{array}{c}
\mathbf{w}_{2}^{H} \\
\hline \vdots \\
\hline \mathbf{w}_{d}
\end{array}\right] \cdot\left[\mathbf{m}_{1}\left|\mathbf{m}_{2}\right| \ldots \mid \mathbf{m}_{d}\right] \\
& =\left[\begin{array}{c|c|c|c}
0 & * & \ldots & * \\
\vdots & \vdots & * & \vdots \\
0 & * & \ldots & *
\end{array}\right]
\end{aligned}
$$

Therefore:

$$
\begin{aligned}
\mathbf{V}_{d-1} & =\mathbf{P}_{d-1}^{H} \mathbf{M}_{d} \mathbf{S}=\mathbf{P}_{d-1}^{H} \mathbf{M}_{d-1} \cdot\left[\frac{\mathbf{s}_{2}}{\frac{\vdots}{\mathbf{s}_{d}}}\right] \\
& =\mathbf{P}_{d-1}^{H} \mathbf{M}_{d-1} \mathbf{S}_{d-1}
\end{aligned}
$$

where $\mathbf{S}_{d-1}$ are the last $d-1$ rows of $\mathbf{S}$, and $\mathbf{M}_{d-1}$ the last $d-1$ columns of $\mathbf{M}$. The last equation demonstrates that we have managed to extract a subspace of 
rank $d-1$ free of signal $\mathbf{s}_{1}$.

Next, we need to prove that $\mathbf{P}_{d-1}^{H} \mathbf{M}_{d-1}$ is a full rank square matrix. $\mathbf{P}$ and $\mathbf{M}$ are full rank square matrices (A1), so the determinant of their product is non-zero. Note that their product is equal to:

$$
\begin{aligned}
& \mathbf{P}^{H} \mathbf{M}_{d}=\left[\begin{array}{c}
\frac{\frac{\mathbf{m}_{1}}{\left\|\mathbf{m}_{1}\right\|}}{\mathbf{w}_{2}^{H}} \\
\hline \vdots \\
\hline \mathbf{w}_{d}
\end{array}\right] \cdot\left[\begin{array}{l|l|l|l}
\mathbf{m}_{1} & \mathbf{m}_{2} & \ldots & \left.\mathbf{m}_{d}\right]
\end{array}\right. \\
& =\left[\begin{array}{c|c|c|c}
\left\|\mathbf{m}_{1}\right\| & * & \ldots & * \\
0 & * & \ldots & * \\
\vdots & \vdots & * & \vdots \\
0 & * & \ldots & *
\end{array}\right] \\
& =\left[\begin{array}{c|rr}
\left\|\mathbf{m}_{1}\right\| & * & \ldots * \\
\hline 0 & & \\
\vdots & \mathbf{P}_{d-1}^{H} \mathbf{M}_{d-1} \\
0 &
\end{array}\right]
\end{aligned}
$$

From the determinant properties [30], the determinant of $\mathbf{P}^{H} \mathbf{M}$ is the product:

$$
\left|\mathbf{P}^{H} \mathbf{M}_{d}\right|=|| \mathbf{m}_{1}|| \cdot\left|\mathbf{P}_{d-1}^{H} \mathbf{M}_{d-1}\right|
$$

as the first determinant and the norm of the vector $\mathbf{m}_{1}$ are non-zero, it implies that the determinant, $\left|\mathbf{P}_{d-1}^{H} \mathbf{M}_{d-1}\right|$ is non-zero as well, and therefore the matrix is full rank, so point $\alpha$ ) is demonstrated.

Point $\beta$ ): Be $\mathbf{u}_{1}$ the row produced by the maximum ratio combining beamformer, then:

$$
\mathbf{u}_{1}=\mathbf{m}_{1}^{H} \mathbf{V}_{d}=\left\|\mathbf{m}_{1}\right\|^{2} \mathbf{s}_{1}+\mathbf{m}_{1}^{H} \mathbf{M}_{d-1} \mathbf{S}_{d-1}
$$

Be $\mathbf{U}$ the compound built as: $\mathbf{U}=\left[\mathbf{V}_{d-1}^{T} \mathbf{u}_{1}^{T}\right]$, then:

$$
\begin{aligned}
& \mathbf{U}=\left[\mathbf{V}_{d-1}^{T} \mathbf{u}_{1}^{T}\right] \\
& =\left[\begin{array}{lll|l}
\mathbf{s}_{2}^{T} & \ldots & \mathbf{s}_{d}^{T} & \mathbf{s}_{1}^{T}
\end{array}\right] \cdot\left[\begin{array}{c|c}
\mathbf{Q}_{d-1}^{T} & \mathbf{m}_{1}^{H} \mathbf{M}_{d-1} \\
\hline 0 \ldots 0 & \left\|\mathbf{m}_{1}\right\|^{2}
\end{array}\right]
\end{aligned}
$$

with:

$$
\mathbf{Q}_{d-1}=\mathbf{P}_{d-1}^{H} \mathbf{M}_{d-1}
$$

From assumption (A4), $\mathbf{S}$ is full rank and the compound term on the right of the equation is as well, then $\mathbf{U}$ is full rank. Performing a Gram-Schmidt orthogonalization on the vectors of $\mathbf{U}$ will effectively remove any contribution of $\mathbf{s}_{2}$ till $\mathbf{s}_{d}$ to the last output vector, which will be orthogonal to them and therefore be proportional to $\mathbf{s}_{1}$ (recall assumption (A3)). Practically, a reduced QR factorization on the matrix $\left[\mathbf{V}_{d-1}^{T} \mathbf{v}_{i}^{T}\right]$ delivers $\mathbf{s}_{1}^{T}$.

Prior to the algorithm, we need to estimate the noise power for the statistical tests. We estimate the noise standard deviation over a time interval which is chosen if 1 ) there is no cross-correlation between channels (e.g. $\left.\left.\mathrm{E}\left\{x_{i}[n] x_{j}[n]\right\}=\delta_{i j}\right), 2\right)$ if each channel output has a zero skewness and kurtosis, and 3) if each channel output has a time auto-correlation approximately equal to a Dirac function of time: $C_{i}(\tau)=\mathrm{E}\left\{x_{i}[n] x_{i}^{*}[n+\tau]\right\}=$ $\delta(\tau)$.

The algorithm follows the next steps:

1. As in the PA, the data is sliced in segments of 400 samples $(8 \mu \mathrm{s})$ (see below for the rationale of this value) on which a SVD is performed (see Figure 9).

2. For each slice, we use a whiteness test [32] to decide if there are 0,1 , or more sources.

(a) if there exists at least one slice with one source, we apply the Proposition III.1 on the slice with the largest first eigenvalue. Go to step 3.

(b) if only slices exist with either zero or more than one source, then we choose among the slices with several sources, the one which has the largest difference between the first and the second singular value. Go to step 3.

(c) if no source is detected, the algorithm stops.

3 . The extracted source by point $(\beta)$ from Proposition III.1 is kept aside, while the estimated residual subspace by point $(\alpha)$ from Proposition III.1 is used to feed step 1.

In the EPA, the segments from step 1) are longer than in the PA to have a better estimate of the singular values in step 2). The choice made in step 2.b) is done on the rationale that the estimation of the main source signature vector is not too heavily impaired by the other weaker sources.

Similar to the PA, the extracted replies feed a conventional receiver. In this case, another benefit is that under some conditions, a conventional decoder may decode two Modes A/C received on a channel. So if the separation fails, it gives an extra opportunity to decode the replies in given cases.

Remark 1: Note that this type of algorithm is not limited to SSR sources, but to any kind of packet-like sources, e.g. pulse interferences such as the Distance Measuring Equipment (DME) signals. It may also be applied to other signal processing problems such as packet separation in wireless ad hoc networks [34].

Remark 2: While not stated explicitly and not needed, the resulting beamformers for the EPA can be obtained by means of the matrices $\mathbf{P}$ derived at each iteration of the algorithm.

\section{A case study}

We will describe the experimental setup in the next section, but let us have a short sight at the case W5 (Figure 3) as it is very illustrative. At first glance this case contains two short Mode S. A closer examination, however, would reveal a hidden Mode A/C, which, by chance, coincides exactly with the preamble of the first source.

This case has the worst behavior from the case set used in [24], [35], where either one antenna conventional receiver or super-resolution receiver are applied. This case presented a high number of Low Confidence Bit (LCB): between 8 and 14; and of course, the last Mode $\mathrm{S}$ and the Mode A/C are not recovered. Therefore our algorithms are needed. 


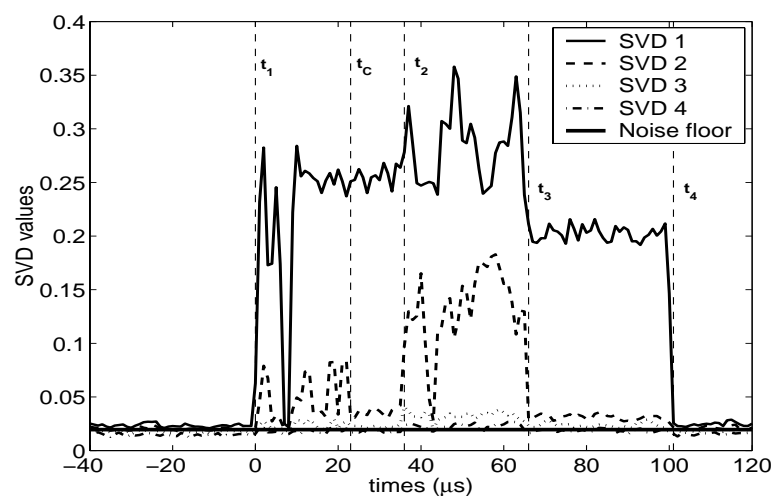

Fig. 9. Detection test over case W5 (time in $\mu \mathrm{s}$ ).

First, let us investigate in Figure 9 the SVD values as a function of time, for detection purpose. The instants $t_{1}$ till $t_{4}$ are shown by means of dotted lines. Note that we also detect the Mode $\mathrm{A} / \mathrm{C}$, which ending time $t_{C}$ is also shown with a dotted line. Therefore, we can assign the number of sources present over each time slot.
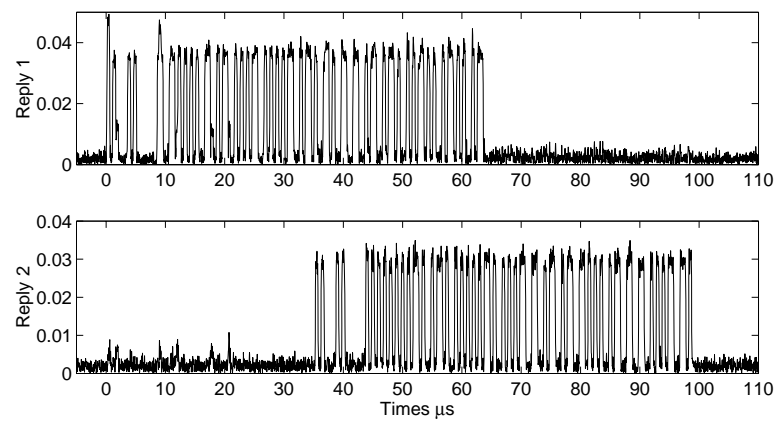

Fig. 10. Case w5: separation achieved by the PA.

Now, we apply our first algorithm, i.e. the PA, to this case. The Mode A/C affects the estimation of $\mathbf{m}_{1}$, but (fortunately) given the ratio of power between the leading Mode S and the Mode $\mathrm{A} / \mathrm{C}$, the error made is of limited extent.

The algorithm estimates well $t_{1}$ and $t_{4}$, the separation is performed and the results are displayed in Figure 10, where the separation is efficient. Indeed we have found that after the PA there remains only one Low Confidence Bit (LCB) for the trailing source, using the conventional decoder. We also note that the Mode A/C is present on both channels, which is reasonable since the PA is not designed to take care of it. On the first channel, the Mode A/C is exactly superposed with the preamble, which is a rare event.

Lastly, we present in Figure 11 the separation result by the Extended PA. By visual inspection, the separation is efficient. By observing the third subplot, one can see that the last pulse of the Mode $\mathrm{A} / \mathrm{C}$ has a large noise peak, which is partly due to a smaller Mode A/C (almost not detectable). This affects the conventional detector, which decides then to reject it; consequently, due to the strict rule we choose, this case will be considered as a failure in later evaluations.
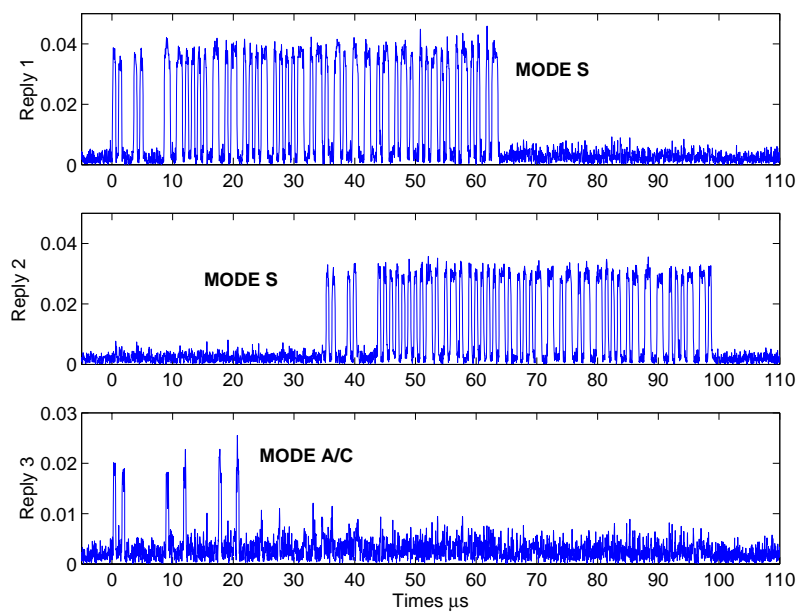

Fig. 11. Case w5: separation achieved by the Extended PA.

\section{Discussion}

Both algorithms are based on projection techniques, but they are aimed at very different situations and they also have an important difference in mandatory requirement. While the PA is conceived to deal with an electromagnetic downlink $(1090 \mathrm{MHz})$ channel situation with Mode S only that is not yet arrived, the EPA is here to solve a situation where the legacy Mode $\mathrm{A} / \mathrm{C}$ is still in the channel. One important difference on the requirement is that the EPA additionally demands that the incoming replies are independent in time. The PA is intrinsically more stable and efficient as it does beamforming based on the space property only, avoiding the Gram-Schmidt step of point $\beta$ in Proposition III.1.

As the EPA is in fact an algorithm that projects out the signal one by one, it can be understood as a technique that would estimate the $\mathbf{m}_{i}$ 's one by one in order to perform a pseudo-inverse at the end, except that this is implicitly done during the algorithm. As the EPA is a projection technique the maximal number of replies that can be separated is equal to the number of antennae.

The theoretical derivation on the angular limitations of PA for an uniform linear array is presented in the Appendix.

Finally, we recall that the state of the art Mode S stations decoder can process only the leading Mode $\mathrm{S}$ reply, and that other separation algorithms would be very expensive [15], [16], or partly or fully fail [7], [9], [10], [11], [18], [14].

\section{EXPERIMENTAL PERFORMANCES}

In this section, we present the performance analysis for both algorithms with the data gathered by the experimental platform that is described in the Appendix. The section is divided in two parts related to PA and EPA, respectively.

The analysis is done using SSR signals received and recorded by means of an ad-hoc system implemented and owned by the Technical University of Delft. This 
system is made up by a four-elements array connected to a wide band digital acquisition system ${ }^{2}$; thanks to it, a large amount of data has been recorded during summer, 2003 [24].

In the first subsection, we study the separation of the mixture of two Mode S by the PA and its competitors. Next, as the EPA algorithm is a global algorithm, we can study the entire data set at our disposal for its evaluation.

\section{A. PA performances}

All the real cases (i.e. 27 recorded signals) have been tested with the projection algorithm. The technique was successful except in two cases, for which the SNR is low $(6$ and $8 \mathrm{~dB})$ and the conditioning number of $\mathbf{M}$ is too high (15 and $12 \mathrm{~dB}$ ), which correspond to an angle difference of $\left(2.17^{\circ}\right.$ and $\left.2.71^{\circ}\right)$ at boresight. On most of these cases the MDA failed due to the too large time delay between two arrivals [12]. The low number of cases in the database of the recorded signals does not allow us to derive statistics for the algorithms, therefore another kind of analysis is required.

Preliminary studies performed at TU Delft on the earlier prototypes have shown that the receivers have a good linearity for the considered dynamic range. Consequently, it is acceptable to consider the addition of two different time slots containing a different Mode $\mathrm{S}$ reply as almost a "real case". The use of these "semisynthesized" cases of overlapping Mode S replies allows us to carry on a general performance analysis of any algorithm on very realistic (not just simulated) signals. This method permits the selection of both the SNR (by adding noise), and the time delay between sources. Furthermore, as we know the individual replies, we also know their message, and can perform a statistical analysis of errors in decoding.

The individual replies were extracted from the measurements and analyzed before being fed to the "synthesis" software; a description is in the Appendix.

We compare our separation algorithm to MDA [5], [16], JADE [11], but also to the conventional decoding algorithm presently used, and to the version with a matched filter presented in [24]. MDA and JADE are both separation algorithms, JADE enforces the statistical independence of the source by using the fourthorder cross-moments of the received signal, while the MDA is a deterministic algorithm that uses the Manchester Encoding of the Mode S replies data block to derive beamformers. In [5], other algorithms, ESPRIT [7], ICA [10], SOBI [18], have been evaluated, but they did not show good performances: for the sake of clarity, we do not present them here.

First, we study the influence of the time delay between the two replies. We keep fixed and equal to 1 the power ratio between sources, and the SNR of each source is equal to $20 \mathrm{~dB}$. For this simulation, we have 294 independent pairs. A failure is declared if one of the two estimated reply messages has more than 24 bits

\footnotetext{
${ }^{2}$ http://cas.et.tudelft.nl/ nicolas/public_exp/
}

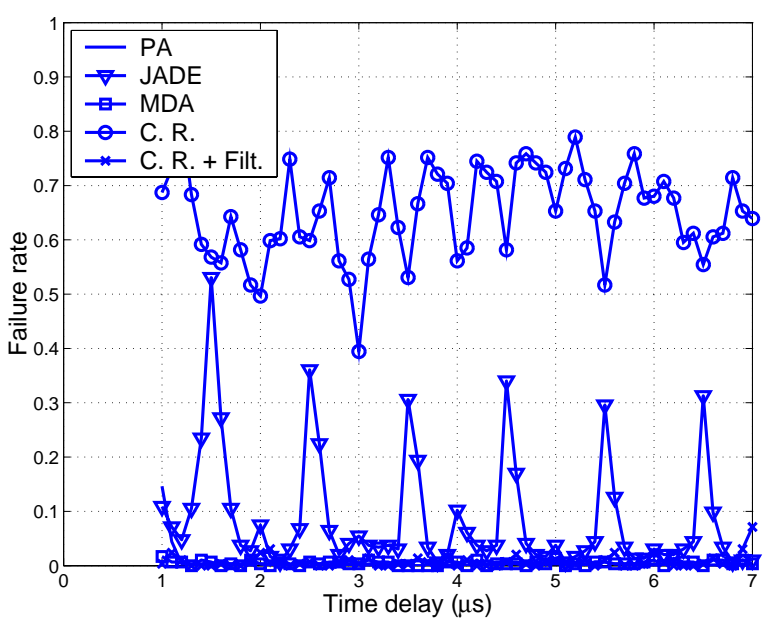

Fig. 12. The failure rate versus the time delay. The PA is represented by the plain curve, while small circles denote the conventional receiver/processor, small crosses its filtered version, small squares the MDA, and triangle the JADE.

wrong, because a redundancy code allows the processor to recover it for a lower number of bit errors. Conventional receivers cannot cope with more than one signal, and therefore the results presented here are for the first signal only. First, note that in Figure 12, JADE has a high failure rate (the reasons can be found in [12]) and therefore, we do not consider it anymore in the following, unless for some comparisons. The conventional receiver (label: $\mathrm{CR}$ ) has a high failure rate as well, this is because this algorithm was not designed to receive two replies at a time. The PA algorithm does not cope for too small delays (below $1.2 \mu \mathrm{s}$ ), which is very reasonable as the algorithm needs the replies not to overlap completely. The failure for the range $[1.5, \ldots, 7] \mu \mathrm{s}$ are of the order of the quantization limit (around 0.003), but their averages over time delay are respectively: 0.0054 for the conventional system with matched filter(label: CR + Filt.), 0.0049 for the MDA, and 0.003 for the PA, which has the best overall rate.

In Figure 13 we present the average errors number per reply versus the time delay, which varies between 1 and $7 \mu \mathrm{s}$. We note that the conventional system not only can decode only one reply, but also has the worst average number of errors per reply. As foreseen, the PA algorithm cannot cope with no time delay, but we note similar performance between the MDA and the PA above $4 \mu \mathrm{s}$, so it appears that it is more appropriate to use the PA from $4 \mu \mathrm{s}$. In [12], the advantage of the PA over the MDA was shown only on simulated data, and it is known [5] that above $40 \mu$ s the MDA is not reliable. The average low confidence bits (LCB) number per reply is not presented here as it has the same shape as Figure 13 and does not bring additional information.

Next, we study the influence of the SNR on the algorithms; the SNR ranges in $[1, \ldots, 24] \mathrm{dB}$, and the time delay is fixed to $6 \mu \mathrm{s}$. The number of independent pairs ranges between 6968 for a SNR of $1 \mathrm{~dB}$, and 12 for $24 \mathrm{~dB}$. 


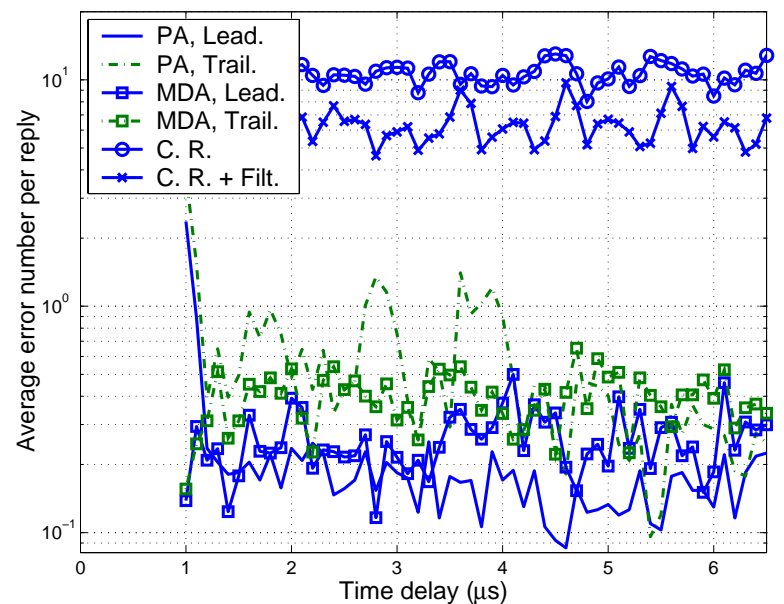

Fig. 13. The average errors number per reply versus the time delay. Small circles denote the conventional receiver/processor, small crosses its filtered version, small squares the MDA, no sign the PA where the plain curves refer to the leading reply, and the dash-dotted to the trailing reply.

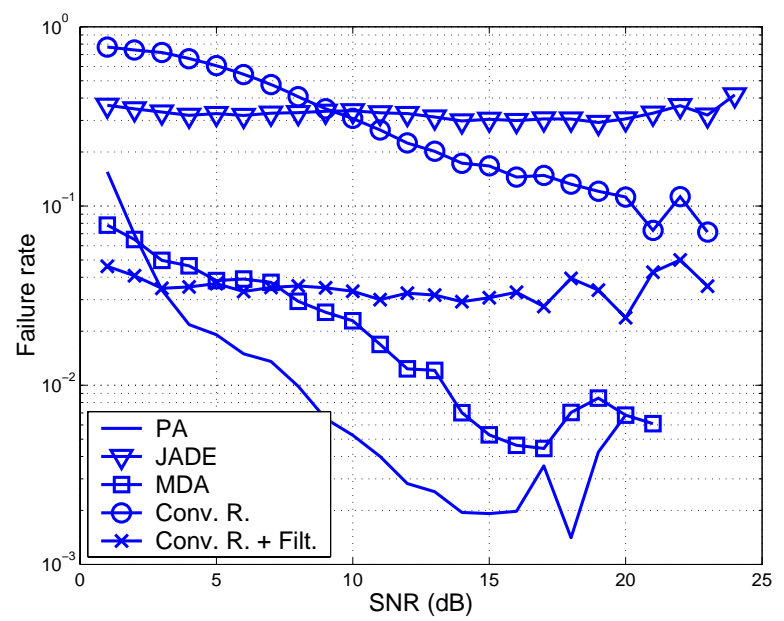

Fig. 14. The failure rate versus the SNR. The PA is represented by the plain curve, while small circles denote the conventional receiver/processor, small crosses its filtered version, small squares the MDA, and triangle the JADE.

From Figure 14, it clearly appears that JADE is not acceptable due to its high error rate. As well, the MDA and the PA algorithm have much better performance than conventional systems. The curves of the MDA and the PA stop before reaching $24 \mathrm{~dB}$, because they don't fail on such a small set (12 pairs). One remarkable point is that the PA has a small loss $(2 \%)$ for SNR values larger than $4 \mathrm{~dB}$, while for a single reply, usual receivers reach their conventional operational limits at SNR values above $10 \mathrm{~dB}$ (or even more), which shows inter alia the $6 \mathrm{~dB}$ gain of a 4-elements array.

In Figure 15, we show the average errors number per reply versus the SNR. Note that both the PA and the MDA have very good behavior as compared to competitors, and follow a nearly linear shape for low SNR. Note that for SNR greater than $21 \mathrm{~dB}$, the average error is equal to zero. The average LCB number per reply is not presented as it does not bring additional

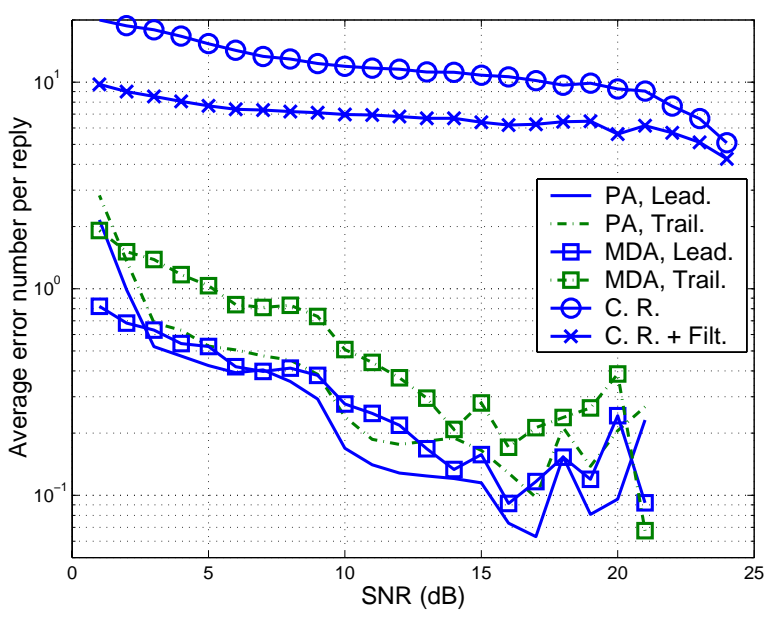

Fig. 15. The average errors number per reply versus the SNR with a time delay of $6 \mu \mathrm{s}$. Small circles denote the conventional receiver/processor, small crosses its matched filtered version, small squares the MDA, no sign the PA; the plain curves refer to the leading reply, and the dash-dotted to the trailing reply.

\section{information.}

In the last experiment, we study the influence of the difference of power between the two sources on the algorithms; the SNR of the trailing source is 15 $\mathrm{dB}$, and the relative SNR of the leading one ranges in $[-10, \ldots, 10] \mathrm{dB}$, i.e. its absolute SNR ranges in $[5, \ldots, 25] \mathrm{dB}$; finally, the time delay is, again, set to 6 $\mu \mathrm{s}$.

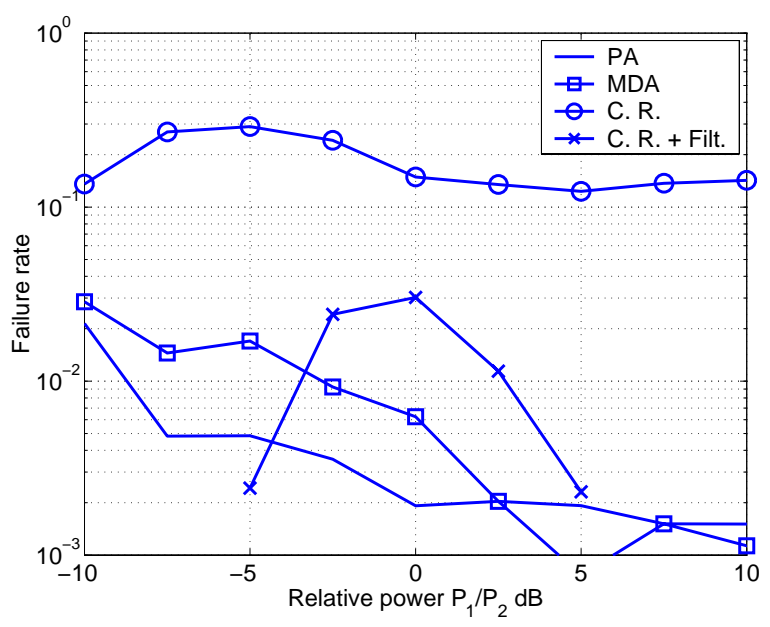

Fig. 16. The failure rate versus the power ratio of the sources. The PA is represented by the plain curve, while small circles denote the conventional receiver/processor, small crosses its filtered version, small squares the MDA, and triangle the JADE.

Figure 16 presents the failure rate versus the ratio between the power of the leading and trailing sources. This is a situation most likely to happen in our problem. First, note that, of course, the central point (0 $\mathrm{dB}$ ) is consistent with the point at $15 \mathrm{~dB}$ in Figure 14. Next, we note that the conventional receiver is equally bad for all situations; but next to it, we have a very interesting discovery, which is that the conventional receiver with a matched filter under-performs 
only for two sources of equal power. Conversely, when one source is largely dominant over the other, it can without failure recover it (nevertheless, it recovers only one). Lastly, we note that the MDA and the PA have a failure rate decreasing as the SNR of the leading source increase, with a similar rate.

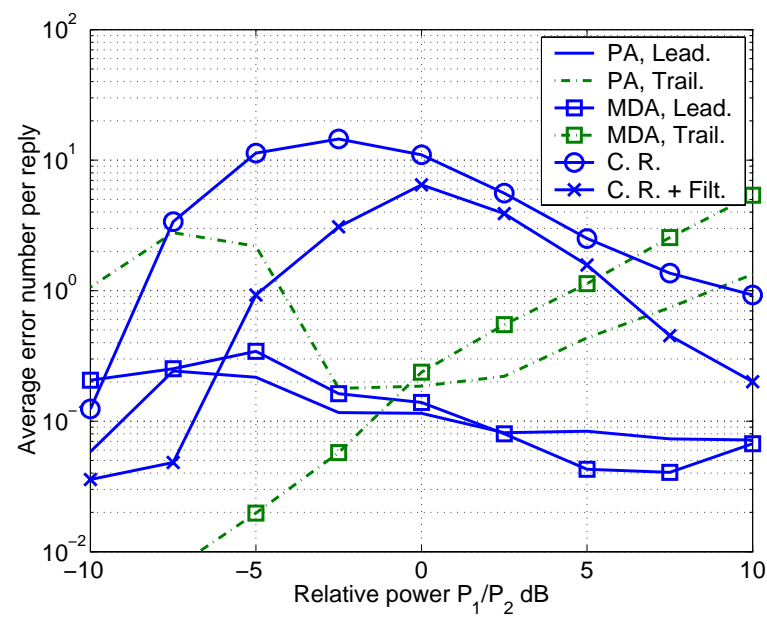

Fig. 17. The average errors number per reply versus the power ratio of the sources with a time delay of $6 \mu \mathrm{s}$. Small circles denote the conventional receiver/processor, small crosses its matched filtered version, small squares the MDA, no sign the PA; the plain curves refer to the leading reply, and the dash-dotted to the trailing reply.

In Figure 17, the average number of errors is presented versus the ratio between the power of the leading and trailing sources. Oppositely to the previous experiments, the average number of errors for the leading and the trailing sources are dissimilar, which makes sense as the SNR are now very much different. These results show the capability of $\mathrm{PA}$ in most cases to correctly decode the leading reply even when its SNR is smaller than the trailing reply SNR, and is as low as 5 or $10 \mathrm{~dB}$.

\section{B. EPA performance}

In this section, as the EPA can handle all cases, we consider all the data measured during summer 2003. The measurement campaign lasted a week, and produced 131 files that have 130000 samples each, i.e. are 2.6 millisecond long. The data were recorded for later off-line processing. We selected the most satisfying records, e.g. from the 3 best days, which represent 70 records.

As the SSR replies are packet-wise, a record is made of several time intervals where a mixture of replies (or a single one) are present, and in-between only noise is present. We cut each record in several cases to keep only the time intervals with replies; in such a way we did obtain 651 different real cases to be processed in our study.

We present in Table I the success rate of our algorithm as compared to a Conventional Receiver. In our experiment, a success is when all replies from a mixture are successfully decoded. Therefore, if one reply is not

\begin{tabular}{|l|c|c|c|c|c|}
\hline Case & $n_{i}$ & $n_{i} / N$ & C.R. & S. & S.+C.R. \\
\hline \hline Global & 651 & 1 & 0.46 & 0.88 & 0.61 \\
\hline \hline Single reply & 303 & 0.47 & 0.93 & 1.00 & 0.94 \\
\hline 2 replies & 190 & 0.29 & 0.07 & 0.90 & 0.39 \\
\hline 2 replies, R.D. & 38 & 0.06 & 0.00 & 0.00 & 0.11 \\
\hline 3 replies, & 84 & 0.13 & 0.00 & 0.93 & 0.29 \\
\hline 3 replies, R.D. & 15 & 0.02 & 0.00 & 0.33 & 0.20 \\
\hline 4 replies & 12 & 0.02 & 0.00 & 0.92 & 0.25 \\
\hline 4 replies, R.D. & 1 & 0.00 & 0.00 & 0.00 & 0.00 \\
\hline More replies & 8 & 0.01 & 0.00 & 0.63 & 0.00 \\
\hline \hline $2 \rightarrow$ 8 replies & 348 & 0.53 & 0.04 & 0.78 & 0.31 \\
\hline \multicolumn{7}{|c|}{ TABLE I }
\end{tabular}

Success TABle: $n_{i}$, Number of EVEnts; $n_{i} / N$, FREQUency OF EVEnts; C.R., SuCCESS RATE FOR the CONVEntional ReCEIVER; S. SEPARATION RATE FOR THE EXTENDED

Projection Algorithm; S. + C.R., success rate for EPA FOllowed By a CONVEntional Receiver. R.D. MEANS "RANK DEFicient" MATRix M.

decoded, we consider it a failure, Table II addresses the failed cases. We classify the various cases by the number of present replies, independently if there are Mode A/C or Mode S. In Table I by denoting Rank Deficient (R.D.), we note that the mixture of the sources has a mixing matrix $\mathbf{M}$ that is rank deficient; for instance two replies impinge from the same direction, the practical limit for the conditioning number being 10 . The Table presents the number of cases for each category, its percentage, then the rate of success for the Conventional Receiver (CR), for the Extended Projection Algorithm (EPA), and for the EPA followed by the CR. A case is a success if all replies are detected (i.e. the Mode A/C brackets are found with their proper characteristics, and the same applies to the Mode S preamble) and sent to the decoding block (whose results, however, cannot be checked due to the lack of knowledge of the particular ICAO transponder code).

We note "a posteriori" that the number of 4 antennae was a good design choice as 4 or more replies occur for only $2 \%$, (although, in airport applications this analysis has to be repeated): having more antennae than sources allows us to perform noise reduction. First, we note that the EPA has an average success rate of only 0.88 . This is due to the fact that in some cases, it was not possible to find a slice of the data containing only one source. The other reason is that the mixing matrix $\mathbf{M}$ is rank deficient or that its conditioning number is high (i.e. (A1) in Proposition III.1 is not respected). We note also that for 3 replies with Rank Deficiency, surprisingly, there are still some successes. In these cases, after separation, there are two output channels, on the first output channel, there is one source, and on the second channel there are the two other replies, but separated in time. A similar effect occurs in the last row of the Table I.

Considering the C.R. success rate for a single reply demonstrates the fact that the C.R. we used is very 
restrictive as it refuses $7 \%$ of the single replies. Due to its design, this receiver has an overall bad performance for a mixture of replies. Because we use it after the separation step, it reduces also the success rate of the full procedure (S.+C.R.). For a single reply, the success rate is almost the same for C.R. and S.+C.R., which is normal since the separation step cannot help for a single reply (apart from the noise reduction). We note that for many replies our success rate is larger by $27 \%$ than the conventional receiver alone. On the overall, the full procedure has a $15 \%$ success improvement over C.R., which mainly obtains one source.

\begin{tabular}{|l|c|c|}
\hline Case & C.R. & S.+C.R. \\
\hline \hline Single reply & 0.00 & 0.00 \\
\hline 2 replies & 0.50 & 0.58 \\
\hline 3 replies, & 0.68 & 1.24 \\
\hline 4 replies, & 0.85 & 2.30 \\
\hline More replies & 1.25 & 2.38 \\
\hline
\end{tabular}

TABLE II

Recovery failure: Average number of Replies DeCoded FOR FAILED CASES FOR the CONVENTIONAL RECEIVER, AND FOR the EPA followed By the CONVENTIONAL ReCEIVER.

As a success in Table I indicates that all replies have been correctly decoded, a failure case may indicate that all replies were lost or only one reply was not decoded. In order to gain some insight on the failed case, we construct the Table II, which presents the average number of replies decoded for the failed cases. The improvement done by the separation step is quite visible in this Table, indeed we note that the effect is not sensible for 2 replies, but it increases in a sensible manner for more replies where two or more replies are recovered.

\section{Conclusion and Perspectives}

In this paper, we have presented several novel solutions to the cumbersome problem of separating SSR replies (Mode A/C and Mode S); i.e. the EPA intended for any number of overlapping Mode S and Mode A/C replies, and the PA, suited to the situation of a pair of overlapping Mode S signals. A Measurement campaign allowed us to analyze many real cases in order to demonstrate that the proposed solutions work satisfactorily in most cases with a significant improvement in decoding performance (and a limited increase in computational cost) with respect to competing solutions.

Possible directions of future research in this area are:

a) Optimization of the array architecture to improve the condition number of the mixing matrix.

b) Self-calibration of the array in order to perform Direction of Arrival measurements.

c) Exploitation of "ad hoc" frequency agility [28] from transponder to transponder.

d) Detection of Mode S preambles alone, so that they could be used when aircraft density is not too high - to update existing tracks, even when the data block with the message and the aircraft Mode S unique address are lost.

e) Time of arrival (TOA) accuracy issues when reply/squitter signals are corrupted by multi-path and, more generally, by overlapping pulses.

\section{ACKNOWLEDGMENT}

The authors wish to thank Selex-Sistemi Integrati and Finmeccanica Group for strongly supporting research activities by Tor Vergata University in the area of SSR by means of the "Centro Ricerche Radar" (CRR). The contribution by Circuits and Systems laboratory (CAS) and the International Research Centre for Telecommunications-transmission and Radar (IRCTR) from Delft Technical University to the acquisition of real SSR signals is also gratefully acknowledged. We would also like to thanks the reviewers for many helpful suggestions aimed to improve the manuscript and N. Niklis for proofreading our manuscript.

\section{REFERENCES}

[1] M. Stevens, Secondary Surveillance Radar. Artech house, Norwood, MA, 1988.

[2] G. Galati, "High precision surveillance system by means of Multilateration of Secondary Surveillance Radar (SSR) signals," 10 May 2005. International Patent Application PCT/IB2005/051519.

[3] G. Galati, P. Magarò, M. Gasbarra, and M. Leonardi, "New signal processing techniques in SSR-Mode S replies multilateration for A-SMGCS," in Proceeding of IRS'04, (Warsaw, Poland), 19-21 May 2004.

[4] P. Bezousek, "A passive radar surveillance system VERA for ATC," in IRS'98, (Munich, Germany), 1998.

[5] N. Petrochilos, "Algorithms for Separation of Secondary Surveillance Radar Replies," phd thesis, Universiy of NiceSophia-Antipolis and TU Delft, Nice, France, July 2002. ISBN 90-407-2371-0, cas.et.tudelft.nl/ nicolas.

[6] R. O. Schmidt, "A Signal Subspace Approach to Multiple Emitter Location and Spectral Estimation," phd thesis, Stanford Univ., Stanford, CA, Nov 1981.

[7] R. Roy and T. Kailath, "ESPRIT estimation of signal parameters via rotational invariance techniques," IEEE Trans. on acoustics, speech, and Signal Processing, vol. 37, pp. 984-995, July 1989.

[8] N. Petrochilos and P. van Genderen, "A new approach to handle SSR replies," in Conférence Radar, (Brest, France), 17-21 May 1999.

[9] E. Chaumette, P. Comon, and D. Muller, "An ICA-based technique for radiating sources estimation; application to airport surveillance," IEE Proceedings - Part F, vol. 140, pp. 395-401, Dec. 1993. Special issue on Applications of High-Order Statistics.

[10] P. Comon, "Independent component analysis, a new concept ?," Signal Processing, Special issue on Higher-Order Statistics, vol. 36, pp. 287-314, April 1994.

[11] J.-F. Cardoso and A. Souloumiac, "Blind beamforming for non-Gaussian signals," IEE Proceedings-F, vol. 140, pp. 362-370, Dec. 1993.

[12] N. Petrochilos and P. Comon, "Separation de signaux ZCM: application en radar SSR," in Proc. of GRETSI 2003, (Paris, France), 8-11 September 2003.

[13] N. Petrochilos and P. Comon, "A zero-cumulant random variable and its applications," Signal Processing Magazine, vol. 86, pp. 3334-3338, November 2006.

[14] Z. Koldovsky and P. Tichavsky, "Efficient variant of algorithm FastICA for independent component analysis attaining the Cramer-Rao lower bound," in IEEE/SP 13th workshop on Statistical Signal Processing, pp. 1090-1095, July 2005.

[15] A. van der Veen and J. Tol, "Separation of zero/constant modulus signals," in Proc. IEEE ICASSP, (Munich, Germany), pp. 3445-3448, April 1997. 
[16] N. Petrochilos and A. van der Veen, "Algorithms to separe overlapping secondary surveillance radar replies," in Proc. of ICASSP 2004, (Montreal, Canada), pp. II.49-53, 17-21 May 2004

[17] N. Petrochilos and P. Comon, "Link between the joint diagonalisation of symmetrical cubes and parafac: an application to secondary surveillance radar," in Proc. of IEEE SAM 2006, (Waltham (MA)), 12-14 July 2006.

[18] A. Belouchrani, K. A. Meraim, J.-F. Cardoso, and E. Moulines, "A blind source separation technique based on second order statistics," IEEE Trans. Signal Processing, vol. 45, pp. 434-444, feb 1997.

[19] N. Petrochilos, G. Galati, L. Mené, and E. Piracci, "Separation of multiple secondary surveillance radar sources in a real environment by a novel projection algorithm," in Proc. of IEEE ISSPIT 2005, (Athens, Greece), 17-21 December 2005.

[20] N. Petrochilos, G. Galati, and E. Piracci, "Projection techniques for separation of multiple secondary surveillance radar sources in a real environment," in Proc. of IEEE SAM 2006, (Waltham (MA)), 12-14 July 2006.

[21] M. Zibulevsky, B. Pearlmutter, P. Bofill, and P. Kisilev, Independent Component Analysis: Principles and Practice, chapter Blind Source Separation by Sparse Decomposition. Cambridge, 2001.

[22] P. Bofill and M. Zibulevsky, "Blind separation of more sources than mixtures using sparsity of their short-time fourier transform," in Proceeding of International Workshop on INDEPENDENT COMPONENT ANALYSIS and BLIND SIGNAL SEPARATION, (Helsinki, Finland), 1922 June 2000.

[23] A. Larue, M. V. D. Baan, J. I. Mars, and C. Jutten, "Sparsity or whiteness: what criterion to use for blind deconvolution of seismic data?," pp. 1642-1645, 2005. SEG Technical Program Expanded Abstracts.

[24] G. Galati, S. Bartolini, and L. Menè, "Analysis of SSR signals by super resolution algorithms," in Proceeding of IEEE symposium ISSPIT-04, (Roma, Italy), Dec. 2004.

[25] R. Trim, "Mode S: an introduction and overview," Electronics \& Communication Engineering Journal, vol. 2, pp. 5359, Apr. 1990.

[26] G. Galati and al., "New approaches to Multilateration processing: Analysis and Field Evaluation," in Proceeding of European Microwave Week, EURAD 06, (Manchester, UK), 13-15 Sept. 2006.

[27] J. E. Hershey, R. T. Hoctor, and all, "Digital Receiving System for Dense Environment of Aircraft," 14 Sep. 2004. USA Patent 6,792,058 B1, by Lockheed Martin Corporation.

[28] G. Galati, "High-capacity location and identification system for cooperating mobiles with frequency agile and time division transponder device on board," 12 October 2005. International Patent Application PCT/IB2005/053343.

[29] G. H. Golub and C. V. Loan, Matrix Computation. Baltimore, MD: Johns Hopkins University Press, 1996.

[30] R. A. Horn and C. R. Johnson, Matrix Analysis. Cambridge University Press, 1985.

[31] International Civil Aviation Organisation, International standards and recommended practices, aeronautical telecommunications: ICAO Doc. 7300/8.I, Annex 10.

[32] S. Haykins and all, Radar Array Processing. SpringerVerlag, 1993.

[33] H. Krim and M. Viberg, "Two decades of array signal processing research," IEEE sig. Proc. Magazine, pp. 67-94, July 1996.

[34] R. Djapic, G. Leus, A. van der Veen, and A. Trindade, "Blind synchronization in asynchronous ultra wideband (UWB) networks based on the transmit-reference scheme," Subm. J. Wireless Comm., Mar. 2005.

[35] G. Galati, E. Piracci, and M. Gasbarra, "Decoding techniques for SSR Mode S signals in high traffic environment," in Proceeding of European Microwave Week, EURAD 05, (Paris, France), 6-7 Oct. 2005.

\section{APPENDIX}

\section{Angular Limitations}

The PA algorithm is based on three essential steps: 1) detection of the number of replies, 2) estimation of the mixing matrix $\mathbf{M}$, and 3 ) inversion of this matrix, steps 1) and 3) depending on the particular mixing matrix. Indeed for a rank-deficient $\mathbf{M}$ (for instance: two replies incoming from nearly the same direction), the detection of the number of replies is inaccurate, and the inversion is not possible. In the following, we analyze both detection and inversion versus the angular separation of two incoming replies.

\section{A. Detection}

One important point in our algorithm is the detection of the presence of two or more sources. If the angles of arrival of two sources are too close, it will be impossible to distinguish that several sources are present. Many tests for the detection of the number of sources are based on the eigenvalues of the covariance matrix, therefore we investigate their values for different angles of arrivals and power levels. In the case of a perfect array, $\mathbf{M}=\mathbf{A G}$, the covariance matrix is equal to:

$$
\mathbf{R}_{x}=\mathrm{E}\left\{\mathbf{x}^{H} \mathbf{x}\right\}=\mathbf{A} \mathbf{R}_{s} \mathbf{A}^{H}+\sigma^{2} \mathbf{I}_{d}
$$

where $\mathbf{R}_{s}$ is the signal covariance matrix of dimension $d \times d$ equal to $\mathbf{R}_{s}=\mathbf{G E}\left\{\mathbf{S S}^{H}\right\} \mathbf{G}^{H}$, and $\mathbf{A}$ the array matrix is of dimension $m \times d$, with usually $d<m$.

By performing the computation of $P_{\lambda}=\left|\mathbf{R}_{x}-\lambda \mathbf{I}_{d}\right|$ in the case of two sources with power $P_{i}$, direction of arrival $\theta_{i}$, and array signature $\mathbf{a}_{i}$, for $i \in\{1,2\}$, we obtain:

$$
P_{\lambda}=\left(\sigma^{2}-\lambda\right)^{m-2} \cdot\left|\begin{array}{cc}
P_{1}+\sigma^{2}-\lambda & \mathbf{a}_{1}^{H} \mathbf{a}_{2} P_{1} \\
\mathbf{a}_{2}^{H} \mathbf{a}_{1} P_{2} & P_{2}+\sigma^{2}-\lambda
\end{array}\right|
$$

where we note that $\sigma^{2}$ is an eigenvalue of order $m-2$, and the calculation of the determinant gives us the two other eigenvalues:

$$
\lambda_{i}=\frac{P_{1}+P_{2}}{2}+\sigma^{2} \pm \sqrt{\frac{\left(P_{1}-P_{2}\right)^{2}}{4}+P_{1} P_{2}\left\|\mathbf{a}_{1}^{H} \mathbf{a}_{2}\right\|^{2}}
$$

then the decision theory makes us compare the result with a threshold: $\lambda_{i} \gtrless c \sigma^{2}$, with $c$ a constant according to the desired false alarm rate.

Figure A1 shows the eigenvalues for a 4-elements antenna array receiving two equi-powered sources, one fixed at the DOA $90^{\circ}$, the other varying, with a SNR equal to $12 \mathrm{~dB}$. The dashed line represents the limit for a false alarm probability of $10^{-6}$ with 100 samples. We note that for such a low SNR if we would use this criterion, it would not be possible to decide that $d=2$ for angular difference less than $12^{\circ}$.

\section{B. Inversion}

Given the model, $\mathbf{X}=\mathbf{M S}+\mathbf{N}$, the pseudo-inverse is then: $\mathbf{M}^{\dagger}=\left(\mathbf{M}^{H} \mathbf{M}\right)^{-1} \mathbf{M}^{H}$; assuming that the estimate $\hat{\mathbf{M}}$ is perfect, the estimated replies are:

$$
\begin{aligned}
\hat{\mathbf{S}} & =\mathbf{M}^{\dagger} \mathbf{X}=\mathbf{M}^{\dagger} \mathbf{M} \mathbf{S}+\mathbf{M}^{\dagger} \mathbf{N} \\
\text { i.e. } \hat{\mathbf{S}} & =\mathbf{S}+\mathbf{M}^{\dagger} \mathbf{N}
\end{aligned}
$$

In order to estimate the output noise power, we have to estimate $\mathbf{M}^{\dagger}$. We consider two sources impinging on 


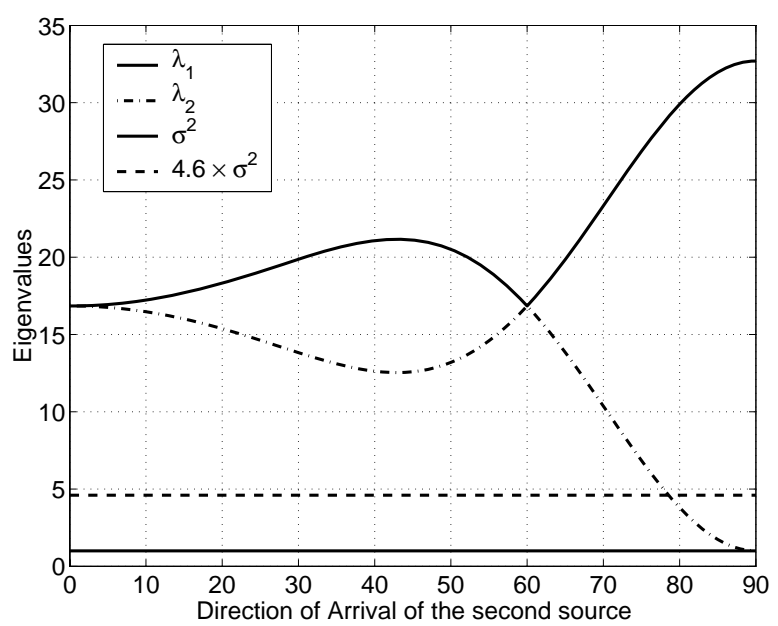

Figure A1: Eigenvalues for the matrix $\mathbf{R}_{x}$, and the

limit for the false alarm rate versus the second direction of arrival (the directions are measured with respect to the array, i.e.to the endfire).

a perfectly calibrated uniform linear array, then, for $m$ elements, the following product stands:

$$
\left(\mathbf{M}^{H} \mathbf{M}\right)^{-1}=\frac{1}{m\left(1-|\alpha|^{2}\right)}\left[\begin{array}{cc}
1 & -\alpha \\
-\alpha^{*} & 1
\end{array}\right]
$$

where $\alpha=\frac{1}{m} \sum_{k=0}^{m-1} a_{2}^{k} a_{1}^{-k}$, with the definition: $a_{i}=$ $\exp \left(\jmath \pi k \sin \theta_{i}\right)$, for $i \in\{1,2\}$ (to keep the presentation simple, we consider here equipowered sources, i.e. $\mathbf{M}=\mathbf{A})$. Denoting at the time $t$, the noise vector $\mathbf{n}[t]=\left[n_{1}[t] \ldots n_{m}[t]\right]^{T}$, the output noise for the separated sources at time $t$ is:

$\left.\mathbf{M}^{\dagger} \mathbf{N}\right|_{[t]}=\left[\begin{array}{c}n_{1}^{o}[t] \\ n_{2}^{o}[t]\end{array}\right]=\left[\begin{array}{c}\sum_{k=0}^{m-1}\left(a_{1}^{-k}-\alpha a_{2}^{-k}\right) n_{k}[t] \\ \sum_{k=0}^{m-1}\left(a_{2}^{-k}-\alpha^{*} a_{1}^{-k}\right) n_{k}[t]\end{array}\right]$

For a spatially white input noise, the power of the output noise is the same for each source and equal to:

$$
\sigma_{\text {out }}^{2}=\frac{1}{1-|\alpha|^{2}} \times \frac{\sigma_{\text {in }}^{2}}{m}
$$

where $|\alpha|^{2}$ is the square of a modified Airy function:

$$
|\alpha|^{2}=\frac{\sin ^{2}\left(\frac{\pi m}{2}\left[\sin \theta_{1}-\sin \theta_{2}\right]\right)}{m^{2} \sin ^{2}\left(\frac{\pi}{2}\left[\sin \theta_{1}-\sin \theta_{2}\right]\right)}
$$

Equation (A1) indicates the noise power after the separation as a function of the input noise power and a function depending of the angles of arrival, that we name "noise gain"; we can use this equation to derive a power budget, as the output SNR will be equal to the input SNR less the noise gain (in $\mathrm{dB}$ ). The minimum output SNR necessary to the preamble detectorestimator and to the conventional decoding function, is $10 \mathrm{~dB}$, then depending on the input SNR, we can have an idea of the closest directions of arrival possible for a good separation.

The noise gain power depends on $\theta_{1}$ and $\theta_{2}$, and cannot be simplified as a function of the difference.

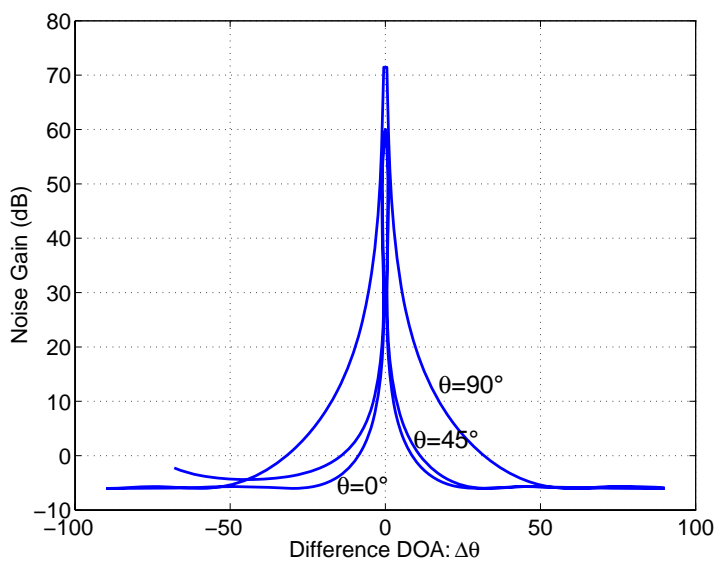

Figure A2: Noise gain as a function of the difference DoA for three different DoA's, $\theta$, of the first source.

For two replies having the same direction of arrival, the gain tends toward infinity. For two directions such that the vectors $\mathbf{a}\left(\theta_{1}\right)$ and $\mathbf{a}\left(\theta_{2}\right)$ are orthogonal, the lowest noise gain is obtained, i.e. $\frac{1}{m}$.

Figure A2 presents the noise gain in $\mathrm{dB}$ for the antenna depending on the Directions of Arrivals of the two replies. Three cases are presented: $\theta_{1}=$ $\left\{0^{\circ}, 45^{\circ}, 90^{\circ}\right\}$, and for each case $\theta_{2} \in\left\{0^{\circ}, 180^{\circ}\right\}$. Because of the end-fire problem, the most relevant case is the boresight direction: $\theta=0^{\circ}$. For instance, in order to separate and decode two equi-powered sources at 20 $\mathrm{dB}$, the two sources need to have a angular difference of $3.5^{\circ}$, or 61 milli-Radians.

\section{EXPERIMENTAL CONSIDERATIONS}

\section{A. Experimental setup}

We describe in this section, the experimental setup we built in CAS/IRCTR from TU Delft to evaluate the separation algorithms with data from real world, i.e. recorded.

The experimental device consists of 4 parts: the antennas, the receiving chain, the digital oscilloscope, and a personal computer (see Figure B1). The impinging signals on the antennas feed the relevant receivers. The receiving chains down-convert the signal from the radio frequency, i.e. nominal $1090 \mathrm{MHz}(\mathrm{RF})$, to the Intermediate frequency, that is set to $10 \mathrm{MHz}$ (IF). They also amplify the signal and filter out the other bands. The digital oscilloscope samples, digitizes and records the output data. Then, via a General Purpose Interface Board link (GPIB), the computer receives the measured data and saves it on the hard-disk and CDROM for off-line processing.

There are 6 antennas (monopoles on a ground plane), and 4 receiving chains. The first and last antenna are loaded with the nominal, $50 \mathrm{Ohm}$, impedance and intended to uniformize the coupling between antennas.

The receiver relevant to each of the four active antennas is also shown in Figure B1. Downstream the antenna, an RF band-pass filter (BP) is placed before the RF-amplifier. Its purpose is to protect the ampli- 


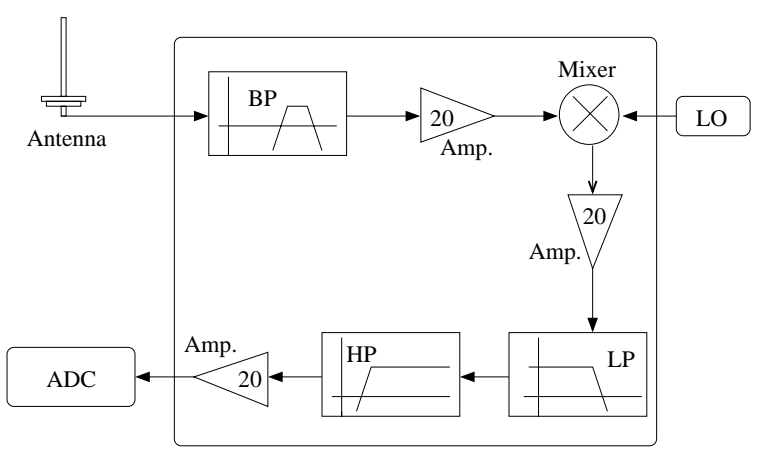

Figure B1: Design of the receiving chain.

fier from increasing the power by other signals. This RF-filter has a bandpass of $10 \mathrm{MHz}$, and is centered at $1090 \mathrm{MHz}$. The amplifier has a gain of $20 \mathrm{~dB}$. The next stage is a mixer, fed by a Local Oscillator (LO). The LO has a frequency of $1080 \mathrm{MHz}$, so the IF will be $10 \mathrm{MHz}$. The LO is from a COTS (Commercial Of The Shelf) waveform generator and is stable in frequency. The next stage consists of a bandpass filter implemented by a LP and a HP filter. The bandpass filter is centered on $10 \mathrm{MHz}$, and has a bandpass of 10 $\mathrm{MHz}$. Measurements made have shown that the filter has a constant time-delay over this bandpass.

The digital part consists of a digital oscilloscope, which is linked to a computer by a GPIB link, and an off-line digital pre-processing phase done in matlab ${ }^{\circledR}$. The oscilloscope, a Tektronix ${ }^{\circledR}$ TDS $\mathbf{7 8 4 A}$, has a vertical resolution of 8 bits. The sampling rate for the measurement is 50 Megasamples per second. As the oscilloscope has a limited memory, it was mandatory to export the data in a fast and reliable way.

The data is loaded from the digital oscilloscope into the computer, which eventually stores it onto CDROM for offline processing. The offline processing consists of three stages. First a Hilbert transform is applied, then a digital filtering with a bandwidth of $10 \mathrm{MHz}$. No synchronization scheme is implemented.

\section{B. Analysis of the single Mode $S$ replies}

In order to create the semi-synthesized data for the evaluation in subsection IV-A, we needed to extract from the data set single Mode S, possibly slightly corrupted with Mode A/C for a more realistic approach. As the measurements were not linked with the Air Traffic Services provider, it is impossible to know the identity, or the position of the transponders. As a consequence, we have to estimate all parameters for each reply (i.e. this is a Blind acquisition).

The first part of this analysis was to remove the replies that did not comply with our requirements. Thus, we removed some incomplete Mode $\mathrm{S}$ as well as the long Mode S (only a few cases).

Lastly, we used a procedure to identify the message contained in the reply (adaptive filter with a stochastic frame on the main SVD eigenvector, followed by a conventional decoder); the procedure was supervised by human expertise (see Figure B2).
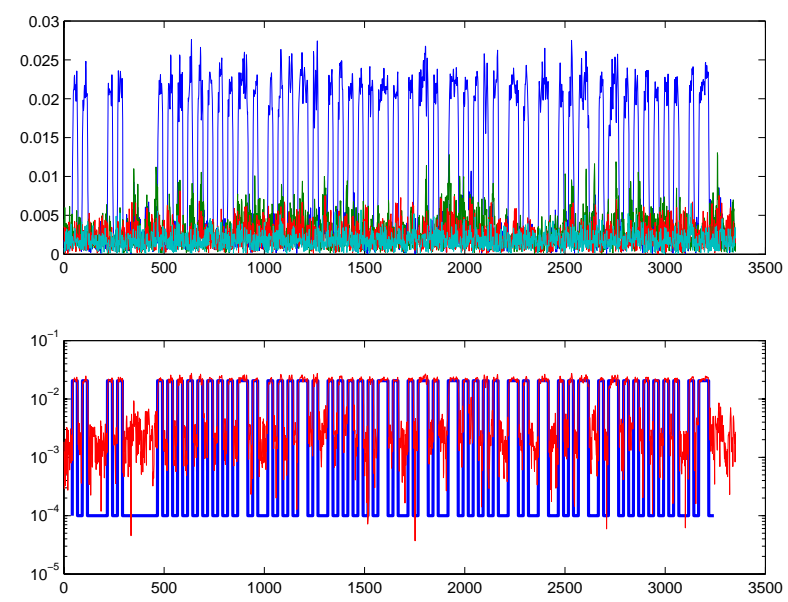

Figure B2: Example of decoding on the case q3, which has an overall SNR of $11.0 \mathrm{~dB}$. Upper plot: Right eigenvectors after the application of a $S V D$.

Lower plot: Received signal compared with the synthesized signal based on estimated parameters.

Figure B2 presents the pre-analysis of the reply q3: in the upper-half, there is the application of SVD to the whole signal obtaining a noise reduction, and separating the signal sub-space (here of dimension one) from the noise sub-space. The lower half of the Figure B2 shows the extracted reply with reduced noise (thin line) and the plot of the estimated reply (thick line). This step is to ensure that the message is well decoded.

When the message could not be validated by the expert, the reply was discarded. We still have an impressive number of replies above $10 \mathrm{~dB}$. Finally, we kept 84 single replies of high quality, for which the message and the other parameters are known. The distribution of those SNR, and their cumulative distribution is presented in Figure B3. We note that on average the SNR is at $16 \mathrm{~dB}$, which is acceptable, as conventional receivers need at least $10 \mathrm{~dB}$, thus it gives us some flexibility during the performance analysis of the PA.

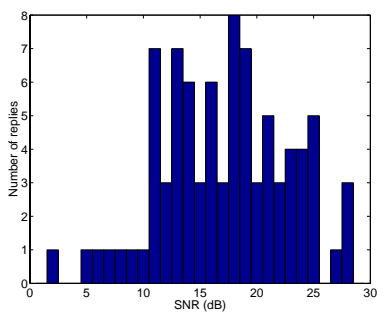

(a) Histogram

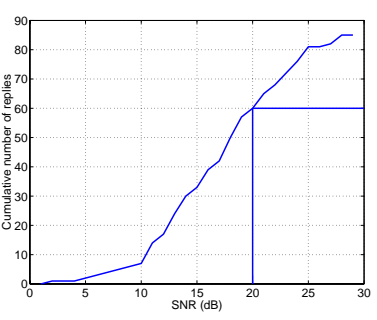

(b) Cumulative histogram
Figure B3: Histogram and cumulative histogram of the SNR of the replies from the main list. 